\title{
Rapamycin upregulates glutamate transporter and IL-6 expression in astrocytes in a mouse model of Parkinson's disease
}

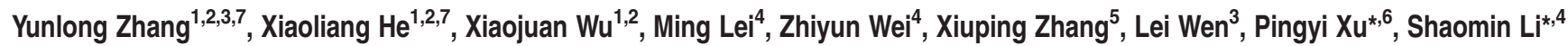 \\ and Shaogang $\mathrm{Qu}^{*, 1,2}$
}

Rapamycin protects mice against 1-methyl-4-phenyl-1,2,3,6-tetrahydropyridine (MPTP)-induced loss of dopaminergic neurons, which is an established model for Parkinson's disease. We demonstrated that rapamycin preserves astrocytic expression of glutamate transporters and glutamate reuptake. The protective effect was also observed in astrocyte cultures, indicating that rapamycin acts directly on astrocytes. In the MPTP model, rapamycin caused reduced expression of the E3 ubiquitin ligase Nedd4-2 (neuronal precursor cell expressed developmentally downregulated 4-2) and reduced colocalization of glutamate transporters with ubiquitin. Rapamycin increased interleukin-6 (IL-6) expression, which was associated with reduced expression of inflammatory cytokines, indicating anti-inflammatory properties of IL- 6 in the MPTP model. NF- $\kappa B$ was shown to be a key mediator for rapamycin, whereas Janus kinase 2, signal transducer and activator of transcription 3, phosphoinositide 3-kinase, and Akt partially mediated rapamycin effects in astrocytes. These results demonstrate for the first time in a Parkinson's disease animal model that the neuroprotective effects of rapamycin are associated with glial and anti-inflammatory effects.

Cell Death and Disease (2017) 8, e2611; doi:10.1038/cddis.2016.491; published online 9 February 2017

The motor symptoms of Parkinson's disease (PD) are predominantly due to the degeneration of dopaminergic (DA) neurons in the pars compacta of substantia nigra (SNpc), ${ }^{1}$ and multiple mechanisms are believed to contribute to neurodegeneration in PD, including $a$-synuclein toxicity, ${ }^{2}$ mitochondrial impairment, ${ }^{3}$ reactive oxygen species, ${ }^{4}$ glutamate excitotoxicity, ${ }^{5-7}$ and increased inflammation. 8 ,9 The 1-methyl-4-phenyl-1,2,3,6-tetrahydropyridine (MPTP) of PD model recapitulates the motor symptoms and preferential cell loss in the SNpc. Its features also include mitochondrial dysfunction, glutamate excitotoxicity, inflammatory response, and so on..$^{10,11}$

Rapamycin inhibits the serine/threonine kinase mTOR (mammalian target of rapamycin) within the mTOR complex 1 , resulting in increased autophagy, and in mice increased lifespan. ${ }^{12}$ Rapamycin has also been verified to be neuroprotective in both $6 \mathrm{OH}$-dopamine and MPTP models of PD, and shows different effects compared with the nonselective mTOR inhibitor torin, because of complete and partial inhibition of mTOR. ${ }^{13}$ A previous study reveals that RTP801(REDD1; Ddit4) is a proapoptotic protein that is sufficient and necessary to induce neuronal death in cellular and animal models of
PD, and rapamycin blocks increased expression of RTP801 in DA neurons and preserves phosphorylation of phosphoinositide 3-kinase at a critical residue. ${ }^{13}$ However, the effects of rapamycin on glia have not been well characterized in animal models of PD.

In this study, we demonstrate that rapamycin directly targets glial cells to limit two well-established contributing factors to PD pathology: astrocyte activation and the inflammatory response. The effects of rapamycin may be mediated via ubiquitin/proteasomal pathways and the modulation of the Janus kinase 2 (JAK2)/signal transducer and activator of transcription 3 (STAT3) pathway. These findings expand our understanding of the protective mechanisms of rapamycin in a PD model and provide additional potential targets for the therapeutic intervention of PD.

\section{Results}

Rapamycin is neuroprotective in the MPTP model of PD. Rapamycin has been shown to protect against neuron death in in vitro and in vivo models of PD. ${ }^{13}$ To verify its

\footnotetext{
${ }^{1}$ Department of Neurology, The First People's Hospital of Shunde Affiliated to Southern Medical University, Foshan, Guangdong 528300, China; ${ }^{2}$ Department of Immunology, School of Basic Medical Sciences, Southern Medical University, Guangzhou, Guangdong 510515, China; ${ }^{3}$ Department of Traditional Chinese Medicine, College of Medicine, Xiamen University, Xiamen Fujian 361102, China; ${ }^{4}$ Ann Romney Center for Neurologic Diseases, Brigham and Women's Hospital and Harvard Medical School, Boston, MA 02115, USA; ${ }^{5}$ Teaching Center of Experimental Medicine, School of Basic Medical Sciences, Southern Medical University, Guangzhou Guangdong 510080, China and ${ }^{6}$ Department of Neurology, The First Affiliated Hospital of Guangzhou Medical University, Guangzhou Guangdong 510080, China ${ }^{*}$ Corresponding author: Shaogang Qu, Department of Neurology, The First People's Hospital of Shunde Affiliated to Southern Medical University, Foshan, Guangdong 528300, China. Tel: +86 757 22318000; Fax: +86 20 61648221; E-mail: sgq9528@ 163.com

or Shaomin Li, Ann Romney Center for Neurologic Diseases, Brigham and Women's Hospital and Harvard Medical School, Boston, MA 02115 , USA. Tel: +1 617 525 5119; Fax: +1 617525 5200; E-mail: shaomin_li@hms.harvard.edu

or Pingyi Xu, Department of Neurology, The First Affiliated Hospital of Guangzhou Medical University, Guangzhou 510080, China. Tel: +86 20 83072726; Fax: +86 20 83062726; E-mail: pingyixujd@ 163.com

${ }^{7}$ These authors contributed equally to this work.

Received 04.7.16; revised 22.11.16; accepted 15.12.16; Edited by A Yaron
} 
a

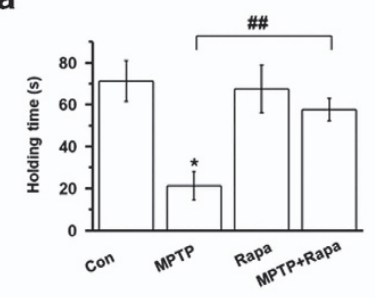

C
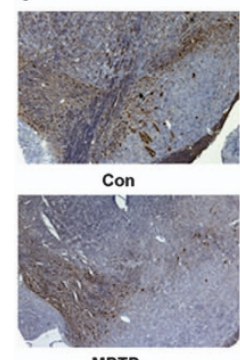

MPTP

e

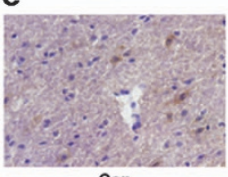

Con

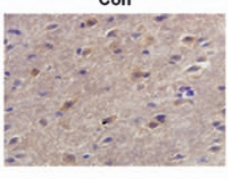

MPTP

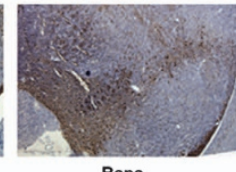

Rapa

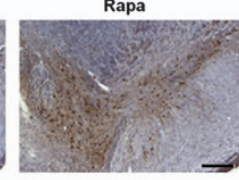

MPTP+Rapa

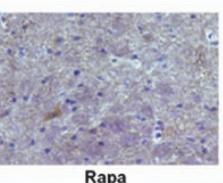

Rapa

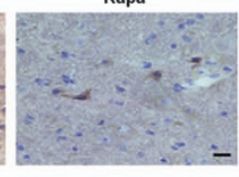

MPTP+Rapa

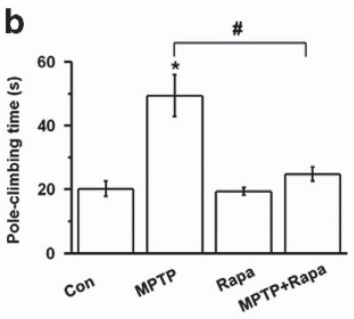

d
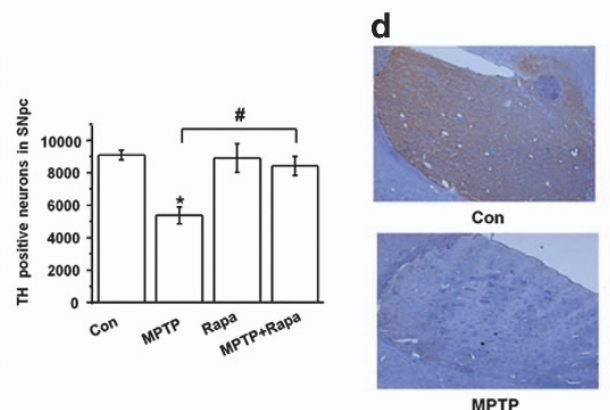

f

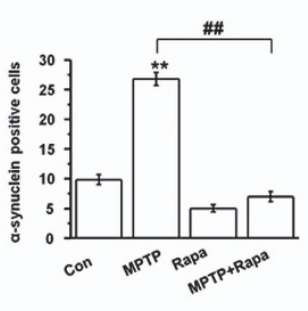

Con

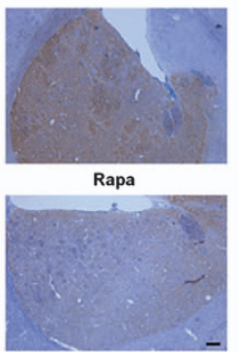

MPTP+Rapa

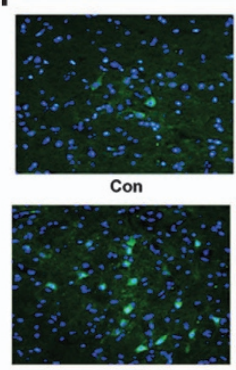

MPTP

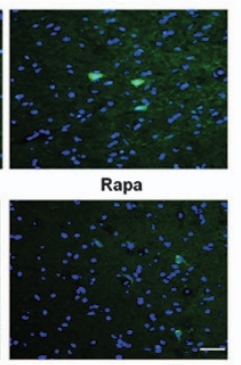

MPTP+Rapa

Figure 1 Neuroprotective effects of rapamycin in MPTP-treated mice. (a and $\mathbf{b}$ ) Behavioral test results of MPTP-treated mice. (a) Grasping test and (b) Pole-climbing test ( $n=12$ per group, one-way analysis variance (ANOVA)). (c and d) IHC of TH in the SNpc (panel c) and striatum (panel d) ( $n=6$ per group, one-way ANOVA). Scale bar, $100 \mu \mathrm{m}$. (e) IHC of $\alpha$-synuclein in the SNpc ( $n=6$ per group, one-way ANOVA). Scale bar, $40 \mu \mathrm{m}$. (f) TUNEL staining of apoptotic neurons in the SNpc ( $n=6$ per group, one-way ANOVA). Scale bar, $50 \mu \mathrm{m}$. Each bar represents the mean \pm S.E.M. of at least three independent experiments. ${ }^{\star} P<0.05$, ${ }^{\star \star} P<0.01$, compared with the control group; ${ }^{\#} P<0.05$, ${ }^{\# \#} P<0.01$, for the MPTP versus MPTP+Rapa groups

protective effects in MPTP model, we assigned mice to four treatment groups: saline+vehicle (Con), saline+rapamycin (Rapa), MPTP+vehicle (MPTP), and MPTP+rapamycin (MPTP+Rapa). Compared with the Con group, MPTP model displayed typical behavioral and histopathological deficits, including reduced holding time, increased climbing time, and marked reduction of tyrosine hydroxylase (TH) expression in the SNpc and striatum (Figures 1a-d). Treatment with Rapa had no effect compared with Con treatment. However, MPTP+Rapa treatment led to a significant reversal of the effects of MPTP on holding time $(P<0.01$, panel a), climbing time $(P<0.05$, panel $b)$, and $\mathrm{TH}$ expression in the SNpc $(P<0.05$, panels $\mathrm{c})$ and striatum $(P<0.01$, panels $\mathrm{d})$. Rapamycin also significantly prevented MPTP-induced $a$-synuclein expression in the SNpc $(P<0.01$, Figure 1e) and prevented MPTP-induced increase in TUNEL-positive cells in the SNpc, indicating prevention of MPTP-induced nigral cell death (Figure 1f). These results verify the protective effects of rapamycin in the MPTP mouse model of PD.

Rapamycin increases the expression and function of glutamate transporters in in vitro and in vivo models of PD. To test if the protective effect of rapamycin in the MPTP model may be explained, in part, by increased glutamate transporter (GLT) expression, antibodies against GLT-1 were used for histology. We showed that MPTP reduced GLT-1 expression in the SNpc, but that reduction was attenuated in the MPTP+Rapa group $(P<0.01)$ (Figure 2a). Rapamycin prevented MPTP-induced reduction of glutamate/aspartate transporter (GLAST) expression in the midbrain and GLT-1 expression in the striatum at the total level $(P<0.05)$ (Figure 2b). Furthermore, rapamycin-mediated preservation of GLT-1 and GLAST in MPTP-treated mice was apparent in plasma membrane-enriched but not in the cytoplasmic protein fractions from the midbrain $(P<0.01)$ (Figure $2 \mathrm{c}$ ). The effects of MPTP and rapamycin on the GLTs in MPTP model were confirmed by quantitative real-time PCR (qRTPCR) (Figure 2d). Within MPTP decreased GLT-1 and GLAST mRNA expression in the midbrain and striatum $(0.69 \pm 0.03$ versus 1 for GLT- 1 mRNA in the midbrain, $P<0.01 ; 0.34 \pm 0.02$ versus 1 for GLAST mRNA in the midbrain, $P<0.01 ; 0.78 \pm 0.02$ versus 1 for GLT-1 mRNA in the striatum, $P<0.05 ; 0.69 \pm 0.02$ versus 1 for GLAST mRNA in the striatum, $P<0.05$, respectively). While rapamycin rescued the decreased GLT-1 and GLAST mRNA expression in the midbrain and striatum $(0.86 \pm 0.04$ versus $0.69 \pm 0.03$ 


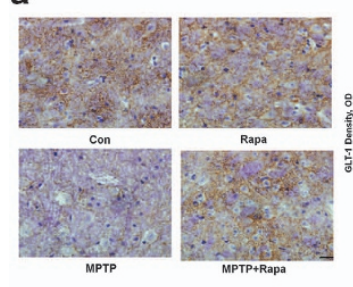

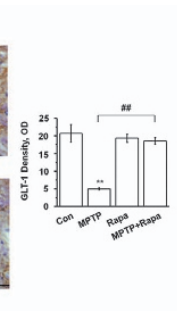
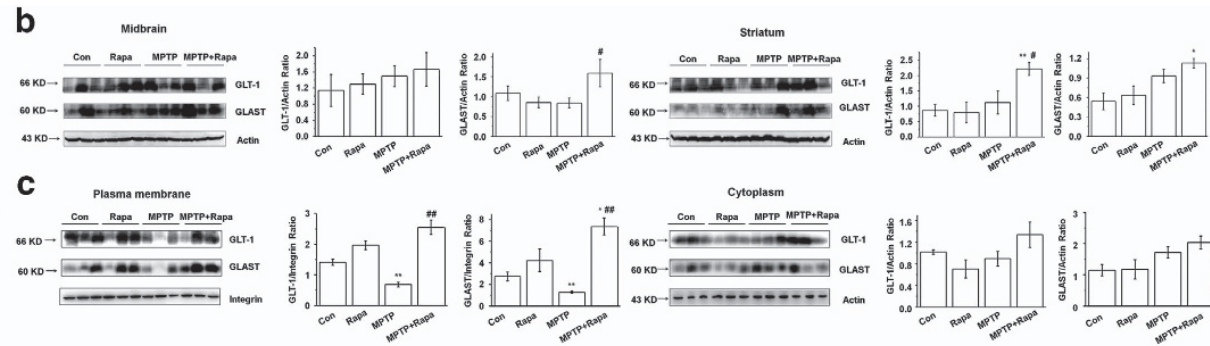

Cytoplasm
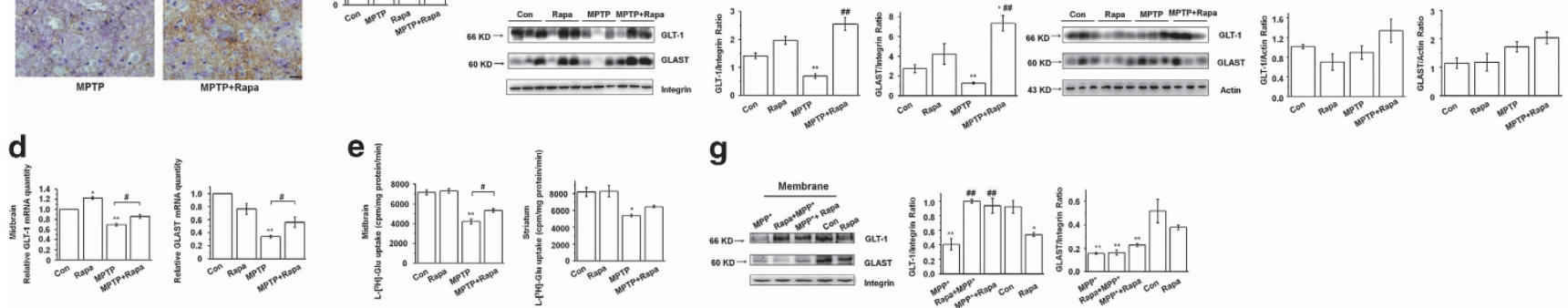

g
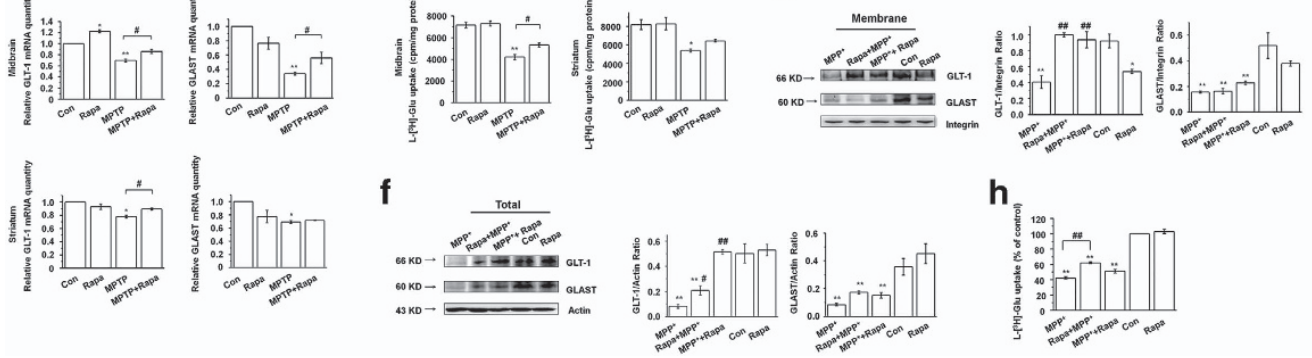

Figure 2 Rapamycin increases the expression and function of GLTs in MPP + -treated astrocytes and in MPTP-treated mice. (a) IHC of GLT-1 expression in the SNpc ( $n=6$ per group, one-way analysis variance (ANOVA)). Scale bar, $40 \mu \mathrm{m}$. (b) Western blotting of GLTs in total protein lysates from the midbrain and striatum ( $n=3$ per group, one-way ANOVA). (c) Western blotting of GLTs in membrane and cytoplasmic preparations from the midbrain and striatum ( $n=3$ per group, one-way ANOVA). (d) qRT-PCR of GLT mRNA expression in the midbrain and striatum ( $n=6$ per group, one-way ANOVA). (e) Glutamate uptake of synaptosomes from the midbrain and striatum ( $n=6$ per group, one-way ANOVA). ( $\mathbf{f}$ and $\mathbf{g}$ ) Western blotting of GLTs in total and membrane protein preparations of astrocytes (at least three experiments, one-way ANOVA). (h) Effects of MPP ${ }^{+}$and rapamycin on glutamate uptake in astrocytes (at least three experiments in triplicate, one-way ANOVA). Each bar represents the mean \pm S.E.M. of at least three independent experiments. ${ }^{*} P<0.05,{ }^{\star \star} P<0.01$ compared with the control group; ${ }^{\#} P<0.05$, ${ }^{\# \#} P<0.01$, for MPTP versus MPTP+Rapa and for MPP ${ }^{+}$versus Rapa+MPP ${ }^{+}$or $\mathrm{MPP}^{+}+$Rapa groups

for GLT-1 mRNA in the midbrain, $P<0.05 ; 0.56 \pm 0.08$ versus $0.34 \pm 0.02$ for GLAST mRNA in the midbrain, $P<0.05$; $0.90 \pm 0.02$ versus $0.78 \pm 0.02$ for GLT-1 mRNA in the striatum, $P<0.05$, respectively). These results indicate that the expression of GLTs was regulated, at least in part, at the transcriptional level by rapamycin. Moreover, the functional effects of MPTP and rapamycin on GLTs were verified by glutamate uptake assays in the midbrain $(P<0.01$ and $P<0.05$, respectively) (Figure 2e).

GLT-1 and GLAST are expressed by astrocytes in the brain; therefore, we speculated that rapamycin may directly target astrocytes to regulate their expression, rather than acting indirectly by inducing prosurvival effects on neurons. To address this possibility, we treated astrocyte cultures with rapamycin and $\mathrm{MPP}^{+}$(1-methyl-4-phenylpyridinium), the active metabolite of MPTP. To account for potential effects of differential order of addition of the drugs, rapamycin was tested both before and subsequent to $\mathrm{MPP}^{+}$treatment. Western blotting of total and plasma membrane lysates demonstrated that rapamycin prevented $\mathrm{MPP}^{+}$-induced decline in GLT-1 expression $(P<0.01$ and $P<0.05$, respectively), but that GLAST expression was not affected by rapamycin cotreatment $(P>0.05)$ (Figures $2 f$ and $g)$. The increased GLT-1 expression was functionally relevant, as indicated by the ability of rapamycin to preserve partially the glutamate reuptake capacity of astrocytes cotreated with MPP $^{+}(P<0.01)$ (Figure 2h).

We previously reported that GLT dysfunction induced by high glutamate levels impairs hippocampal long-term potentiation (LTP). ${ }^{14,15}$ Thus, we perform further experiments in the hippocampus to assess the neuroprotective effects of rapamycin on the PD model. GLT-1 and GLAST levels were significantly increased in the hippocampus after rapamycin administration, and rapamycin partially restores the MPTPinduced decrease in GLT-1 (Supplementary Figure S1a). Additionally, GLT-1 and GLAST mRNA expression in the hippocampus was decreased in MPTP-treated mice and partially restored in MPTP+Rapa-treated mice (Supplementary Figures S1b and S1c). We then examined the effects of MPTP on hippocampal synaptic plasticity in acute brain slices. In contrast to the results of a previous study in which $25 \mu \mathrm{M}$ MPTP increased the baseline in an unsubmerged recording chamber, ${ }^{16}$ we demonstrated that $25 \mu \mathrm{M}$ MPTP did not increase the baseline and instead partially blocked the LTP in a submerged chamber (MPTP: $132 \pm 4 \%, n=6$ versus vehicle: $158 \pm 6 \%, \quad n=8, \quad P<0.01$ ) (Supplementary Figure S1d). Interestingly, $200 \mathrm{nM}$ rapamycin alone had no effect on the LTP $(152 \pm 7 \%, n=8)$, but this low dose of rapamycin could prevent the effects of MPTP on the LTP $(160 \pm 9 \%, n=8)$ (Supplementary Figure S1e). Thus, rapamycin also modulates GLT expression in the hippocampus and prevents MPTP-induced synaptotoxicity.

Rapamycin decreases the ubiquitination of GLTs in the MPTP model. To explore ubiquitination as a potential mechanism contributing to the rapamycin-induced preservation of GLT, we performed immunofluorescence double staining assays for SNpc sections. MPTP increased the colocalization of GLT-1 with ubiquitin, but this colocalization was reversed by MPTP+Rapa treatment (Figure 3a). Interestingly, MPTP+Rapa treatment also reduced GLAST colocalization with ubiquitin, although neither compound 
alone had a significant effect on colocalization (Figure 3b). These findings suggest that rapamycin can inhibit MPTPinduced ubiquitination of GLT-1, and potentially GLAST.
The E3 ubiquitin ligase Nedd4-2 (neuronal precursor cell expressed developmentally downregulated 4-2) has been implicated in the regulation of astroglial glutamine transporters
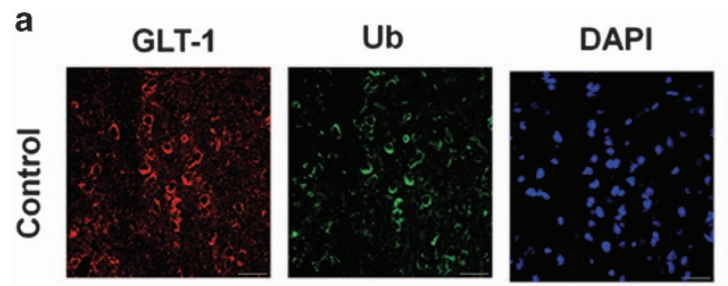

Merge
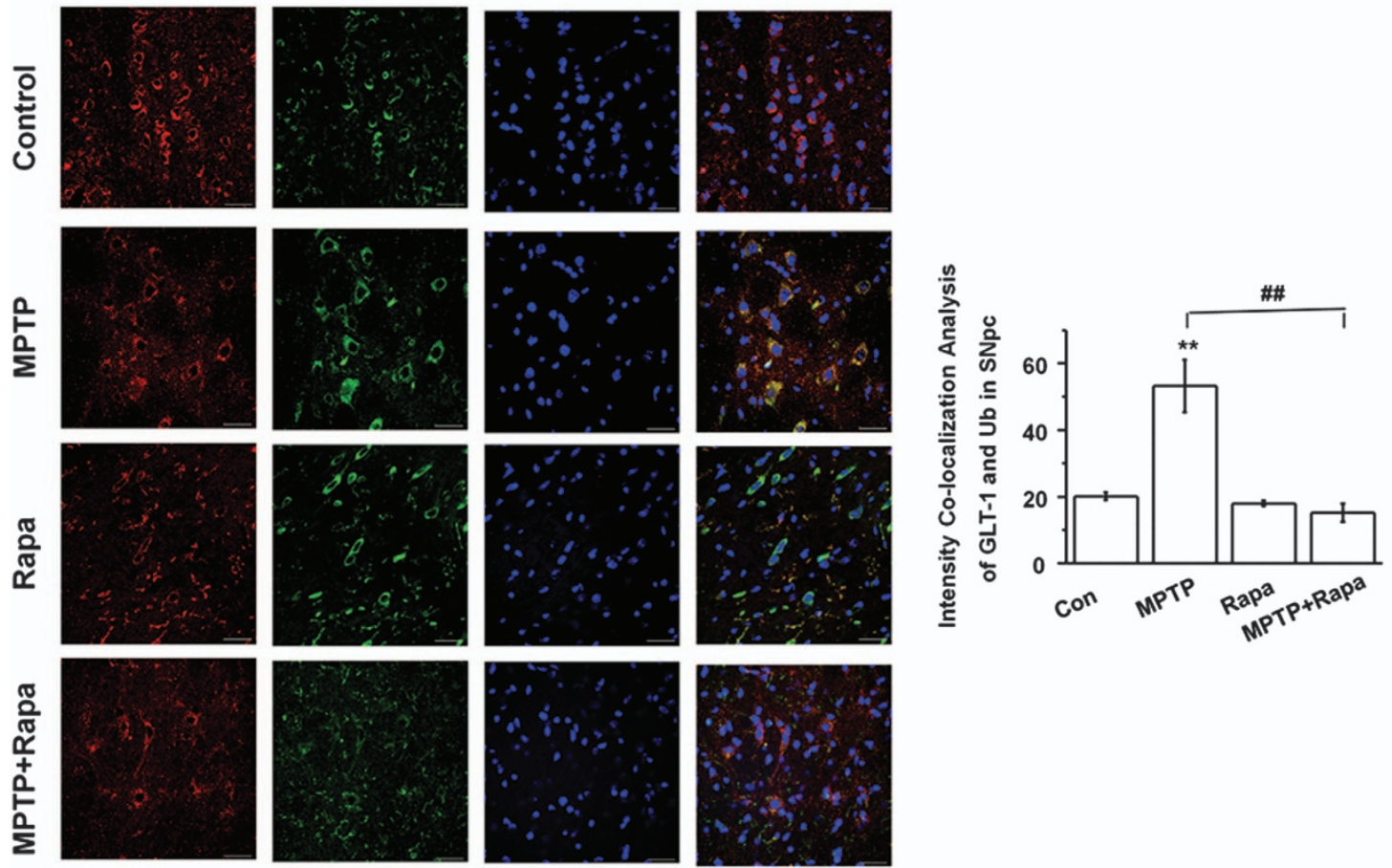

b GLAST

Ub
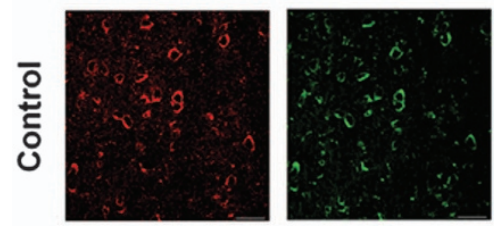

DAPI
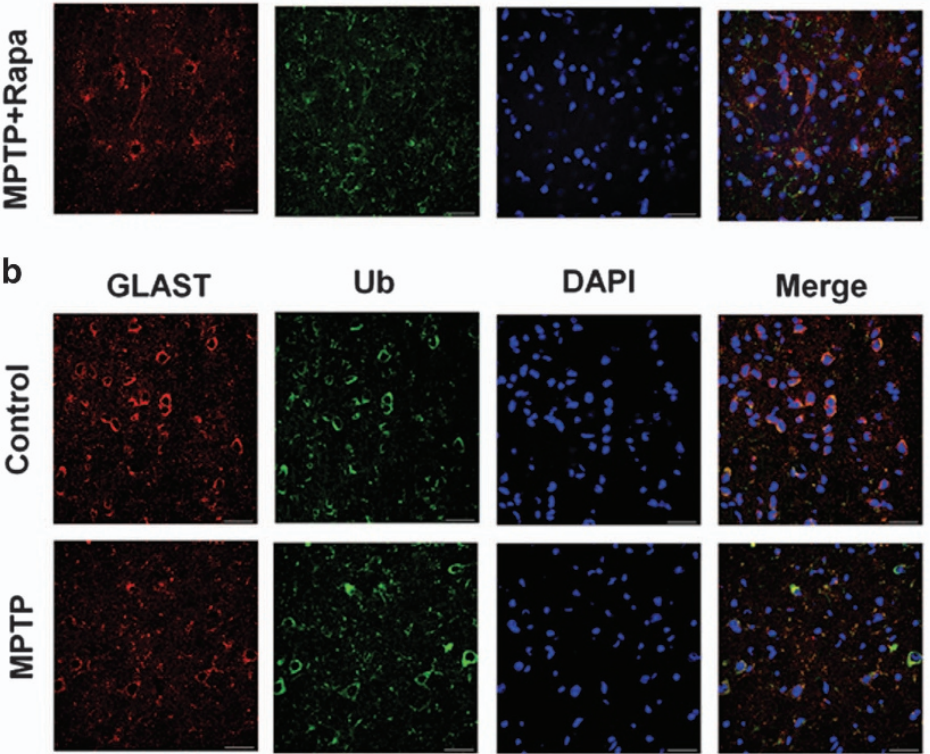

Merge
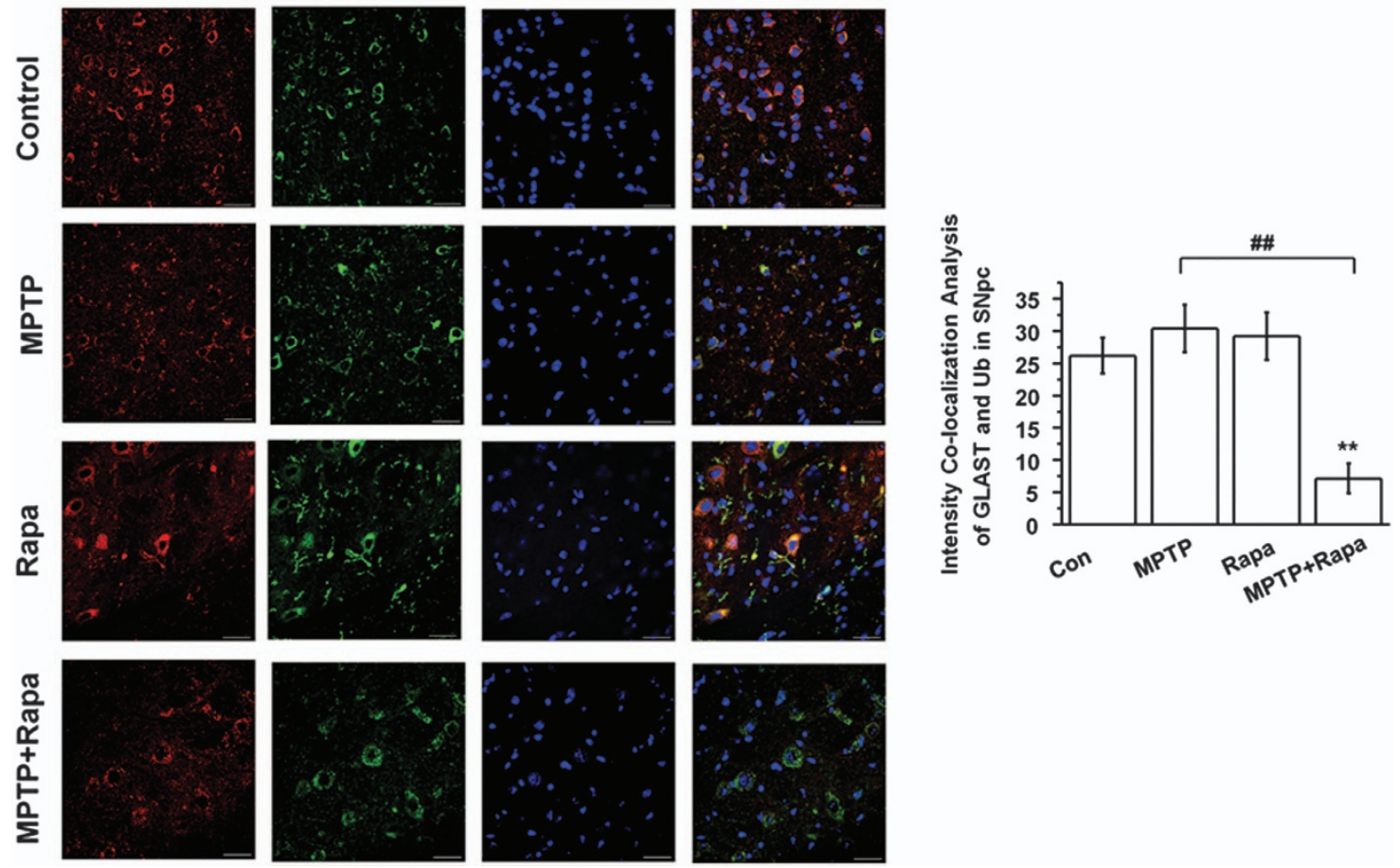

Figure 3 Rapamycin decreases the ubiquitination of GLTs in MPTP-treated mice. ( $\mathbf{a}$ and $\mathbf{b}$ ) Colocalization of ubiquitin and GLTs in the SNpc was assessed by immunofluorescence ( $n=6$ per group, one-way analysis variance (ANOVA)). Scale bar, $30 \mu \mathrm{m}$. Each bar represents the mean \pm S.E.M. of at least three independent experiments. ${ }^{\star *} P<0.01$, compared with the control group; ${ }^{\# \#} P<0.01$ for MPTP versus MPTP+Rapa groups 
a

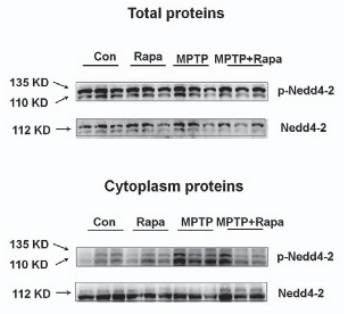

b
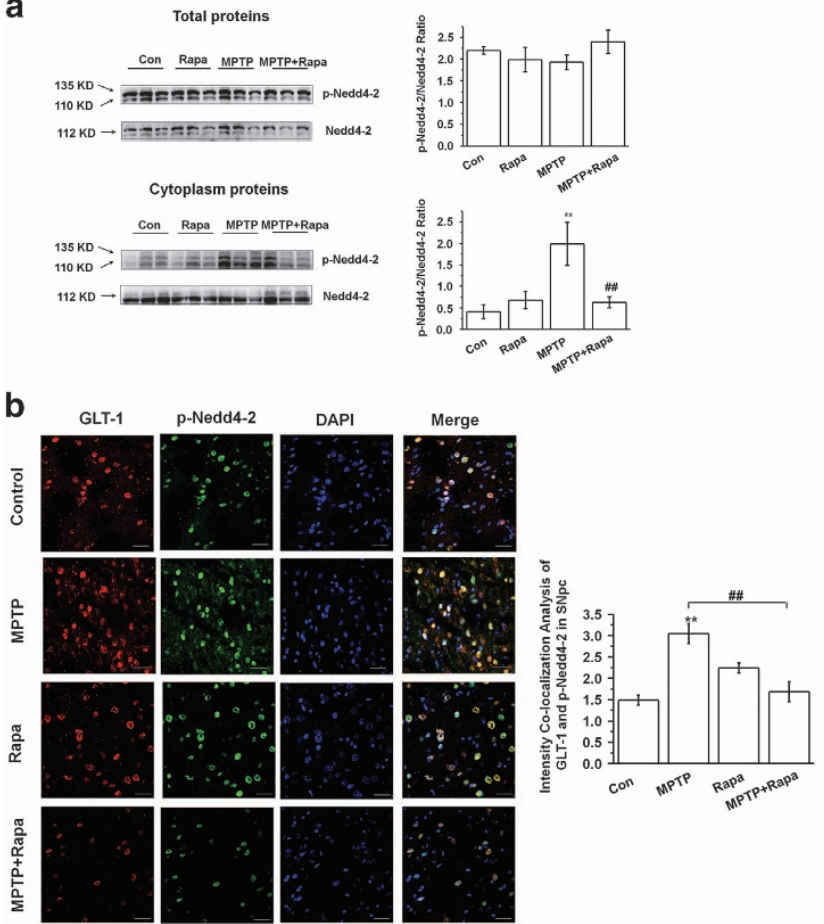

C

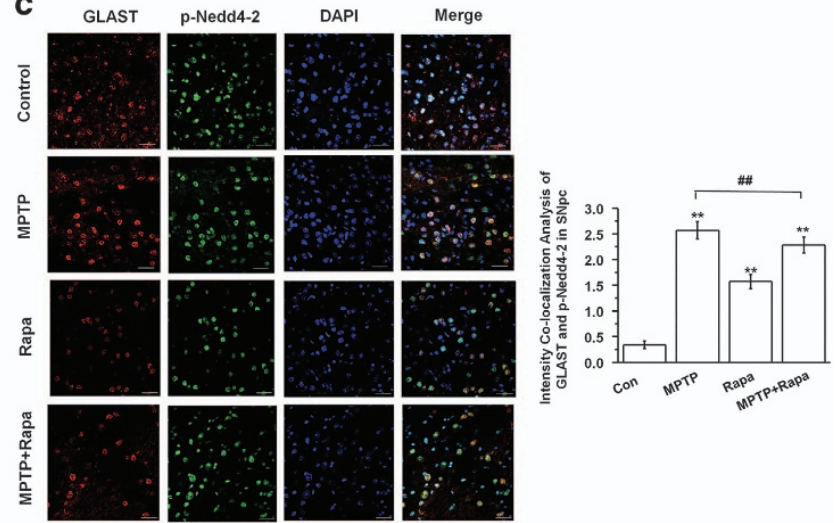

Figure 4 Decreased ubiquitination of GLTs in the treatment of rapamycin is mediated by Nedd4-2 in MPTP-treated mice. (a) Western blotting of phospho (p)-Nedd4-2 and Nedd4-2 in total and cytoplasmic protein preparations ( $n=3$ per group, one-way analysis variance (ANOVA)). ( $\mathbf{b}$ and $\mathbf{c}$ ) Colocalization of $p$-Nedd4-2 with GLTs was determined by immunofluorescence $\left(n=6\right.$ per group, one-way ANOVA). Scale bar, $30 \mu \mathrm{m}$. Each bar represents the mean \pm S.E.M. of at least three independent experiments. ${ }^{* *} P<0.01$, compared with the control group; ${ }^{\# P}<0.01$ for MPTP versus MPTP+Rapa groups

after manganese treatment. $^{17}$ Therefore, we assessed whether MPTP and rapamycin regulates Nedd4-2 activity. We show that MPTP increased the cytoplasmic expression of phosphorylated Nedd4-2 and its colocalization with GLT-1 as assessed by both immunofluorescence staining and western blotting $(P<0.01)$. Furthermore, the increase was prevented by rapamycin cotreatment $(P<0.01)$ (Figures $4 \mathrm{a}$ and $\mathrm{b})$. MPTP also led to increased colocalization of GLAST and Nedd4-2 $(P<0.01)$, whereas rapamycin significantly reduced this colocalization $(P<0.01)$ (Figure 4c). Accordingly, we speculate that rapamycin may reverse the effects of MPTP on GLTs by inhibiting Nedd4-2-dependent ubiquitination.

Rapamycin promotes IL-6 secretion and expression, and activates the JAK2/STAT3 pathway in MPP ${ }^{+}$-treated astrocytes. Interleukin (IL-6) is a major immunomodulatory cytokine with both pro- and anti-inflammatory activities in the pathogenesis of neurodegenerative diseases. ${ }^{18-21}$ It has also been reported to have protective effects in vitro against $\mathrm{MPP}^{+}$-induced neurotoxicity. ${ }^{22}$ Therefore, we investigated the effect of rapamycin on IL-6 in MPP ${ }^{+}$-treated astrocytes. In both pre- and postrapamycin treatment paradigms, the combination of rapamycin and $\mathrm{MPP}^{+}$over $\mathrm{MPP}^{+}$alone induced significantly greater IL-6 expression and secretion (Figures $5 \mathrm{a}$ and $\mathrm{b}, P<0.01$ ). This elevated IL-6 expression was accompanied by a decreased IL-6R expression, which potentially could be a compensatory mechanism $(P<0.05)$ (Figure 5a, second row). Moreover, pre-treatment with rapamycin before $\mathrm{MPP}^{+}$increased the phosphorylation levels of JAK2 and STAT3 $(P<0.01)$, which indicates activation of the JAK2/STAT3 signaling cascade. The effect was significant in the pre-treatment paradigm but was less robust and did not reach statistical significance in the post-treatment paradigm (Figure 5 a, last 5 rows). IL- 6 expression and activation of JAK2/STAT3 pathway were not detected when rapamycin was applied to astrocytes after $48 \mathrm{~h}$ of treatment with MPP ${ }^{+}$ (Supplementary Figure S2a). Thus, in subsequent experiments, we treated astrocytes with $\mathrm{MPP}^{+}$for $24 \mathrm{~h}$.

IL-6 secreted from microglia contributes to the inflammatory reaction in $\mathrm{PD} .^{23-25}$ Here we found that rapamycin did not significantly change the expression of IL-6, IL-6R, or gp130 in $\mathrm{MPP}^{+}$-treated microglial BV2 cells $(P>0.05)$ (Figure $5 \mathrm{c}$, first 3 rows). Furthermore, consistent with previous evidence, ${ }^{26}$ $\mathrm{MPP}^{+}$increased the secretion of IL-6 in BV2 cells, and this increase was blocked by rapamycin in both pre- and posttreatment paradigms $(P<0.05$ and $P<0.01$, respectively) (Figure $5 d$ ). Rapamycin also decreased the expression of phospho-JAK2 and phospho-STAT3 in MPP ${ }^{+}$-treated microglial BV2 cells $(P<0.05)$ (Figure $5 \mathrm{c}$, last 5 rows). This suggests that, unlike in astrocytes, in $\mathrm{MPP}^{+}$-treated microglial BV2 cells rapamycin suppresses IL-6 and the JAK2/STAT3 pathway.

A previous study reported an $\mathrm{MPP}^{+}$-induced decrease of IL-6 mRNA expression in PC12 cells (a cellular model of DA neurons), thereby contributing to $\mathrm{MPP}^{+}$-induced cell death. ${ }^{27}$ Here we confirm that IL-6 expression is decreased in $\mathrm{MPP}^{+}$treated PC12 cells $(P<0.01)$. Additionally, the general phosphorylation status of JAK2/STAT3 pathway was low, and rapamycin had no obvious effects on JAK2/STAT3 phosphorylation in $\mathrm{MPP}^{+}$-treated $\mathrm{PC} 12$ cells $(P>0.05)$ 
a

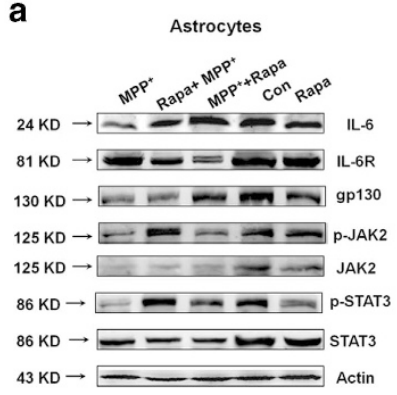

C

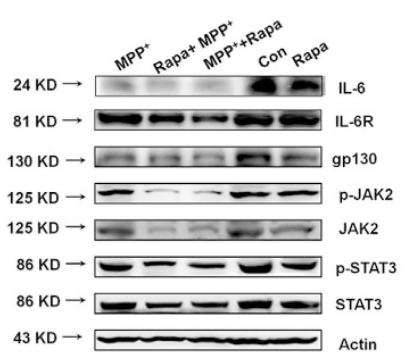

e

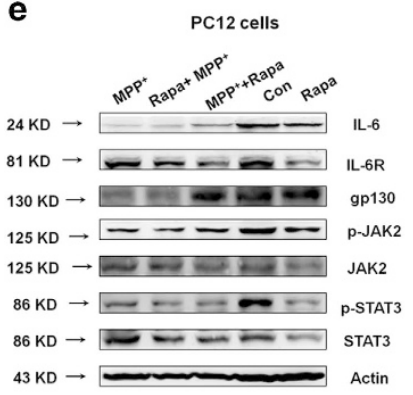

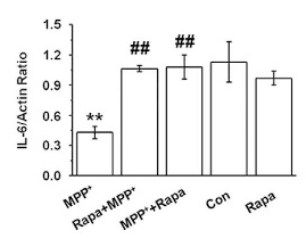
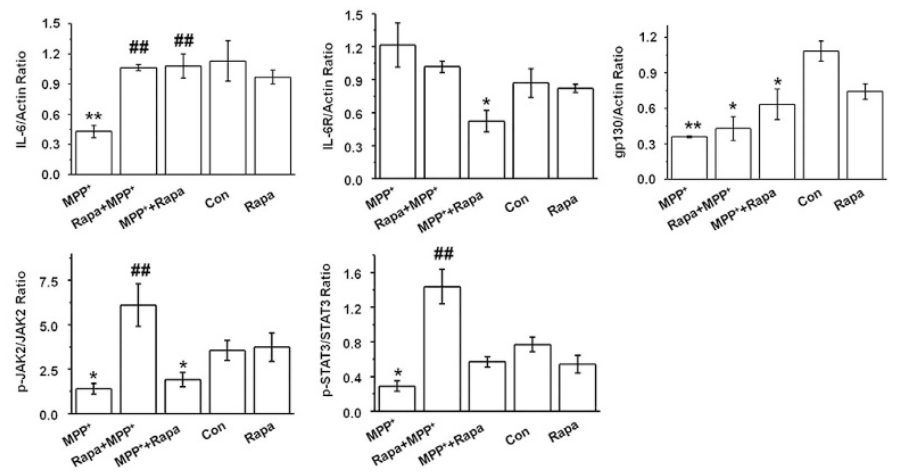

b

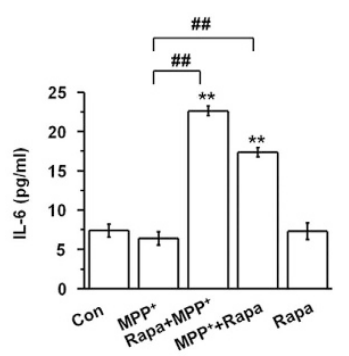

d
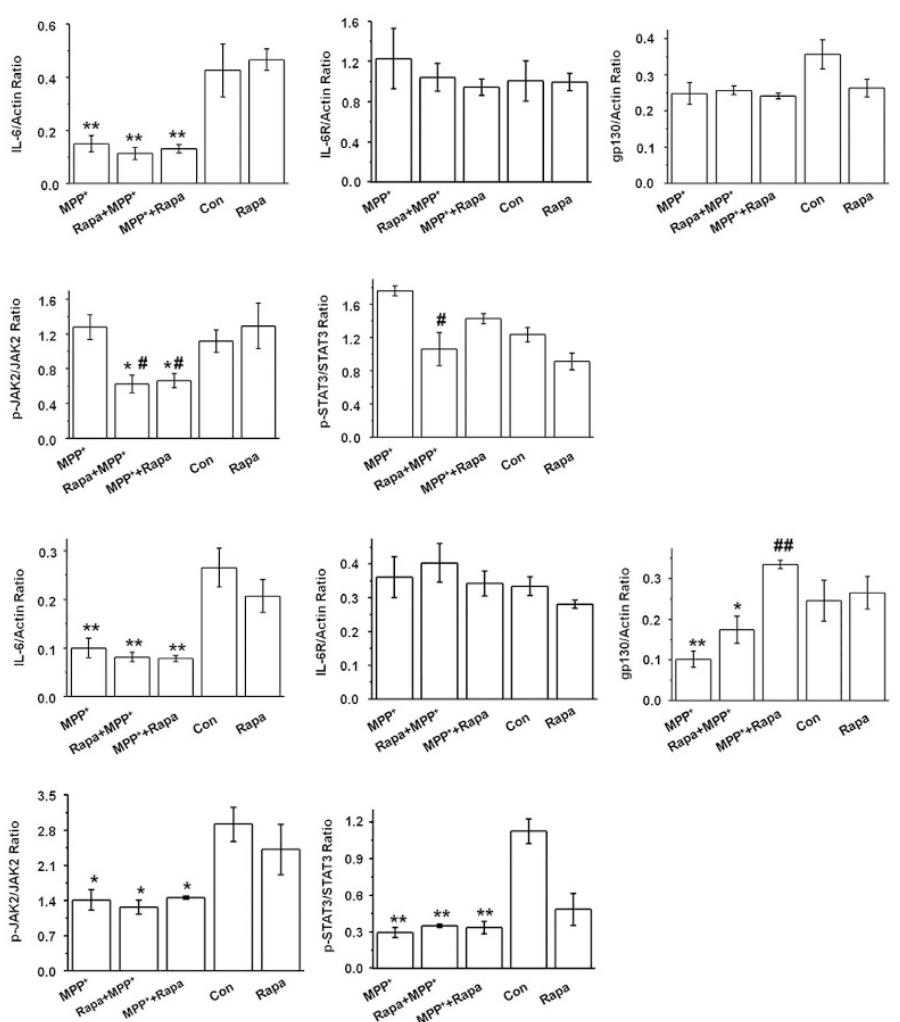

Figure 5 Rapamycin promotes astrocyte-derived IL-6 secretion, increased IL-6 expression and downstream activation of JAK2/STAT3 in MPP'-treated astrocytes. (a) Western blotting of the IL-6/JAK2/STAT3 pathway, IL-6R and gp130 (at least three experiments, one-way analysis variance (ANOVA)). (b) ELISA of IL-6 expression in the astrocyte culture supernatant (at least three experiments in triplicate, one-way ANOVA). (c) Western blotting of IL-6/JAK2/STAT3, IL-6R, and gp130 in BV2 cells (at least three experiments, one-way ANOVA). (d) ELISA of IL-6 expression in microglial BV2 cell culture supernatants (at least three experiments in triplicate, one-way ANOVA). (e) Western blotting of the expression of IL-6/JAK2/STAT3 pathway, IL-6R and gp130 following application of rapamycin in MPP+'treated PC12 cells (at least three experiments, one-way ANOVA). Each bar represents the mean \pm S.E.M. of at least three independent experiments. ${ }^{*} P<0.05,{ }^{\star *} P<0.01$, compared with the control group; ${ }^{\#} P<0.05$, ${ }^{\# \#} P<0.01$, for $\mathrm{MPP}^{+}$versus Rapa+MPP+ or $\mathrm{MPP}^{+}+$Rapa groups

(Figure 5e). These findings suggest that activation of IL-6/ JAK2/STAT3 pathway upon rapamycin/MPP ${ }^{+}$combination treatment may be limited to astrocytes.

Rapamycin promotes astrocyte-derived IL-6 expression in the MPTP model. We further explored whether this phenomenon can be replicated in MPTP-treated mice. Rapamycin was found to significantly increase IL-6 concentrations in the serum of control and MPTP-treated mice $(P<0.01)$ (Figure 6a). IL-6 protein and mRNA expression were also increased in MPTP+Rapa versus MPTP-treated mice in the $\mathrm{SNpc}$ as assessed by immunohistochemistry (IHC) (Figure 6b, $P<0.05$ ) and in the midbrain as assessed by qRT-PCR $(0.88 \pm 0.03$ versus $0.72 \pm 0.04, P<0.05)$ (Figure $6 \mathrm{c}$ ), but this increase was not observed in the striatum $(P>0.05)$ (Figure $6 \mathrm{~d})$. However, rapamycin did not affect the phosphorylation status of JAK2/STAT3 pathway in the midbrain or striatum of MPTP-treated mice $(P>0.05)$ (Figures $6 e$ and f).

To examine the cellular source of increased IL-6 levels in the CNS, we found that rapamycin increased IL-6 expression specifically in astrocytes in Rapa versus Con mice and MPTP 


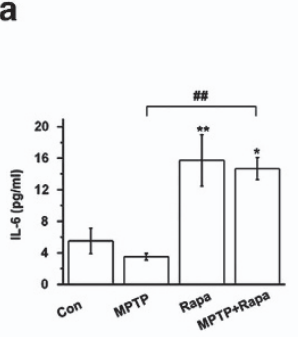

b

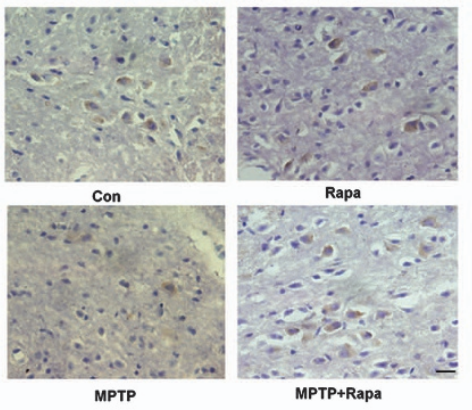

e
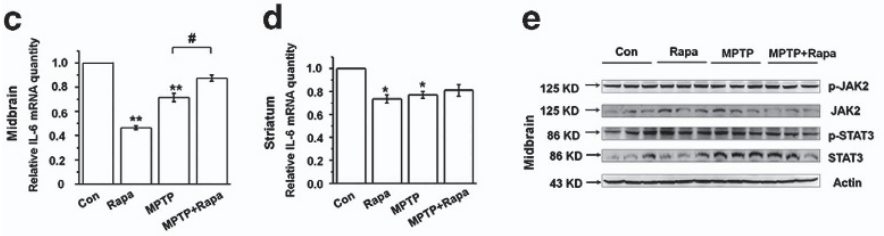
$06 \mathrm{KD} \rightarrow=-\pi=\mathrm{men}=$ $43 \mathrm{KD} \rightarrow \square$ Acti
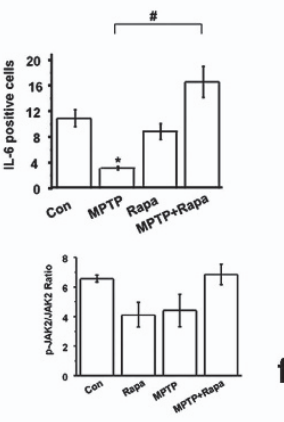

f

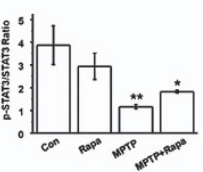

Con Rapa MPTP MPTP+Rapa

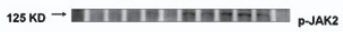

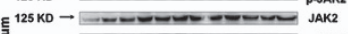
$86 \mathrm{KD} \rightarrow$ ${ }_{436 \mathrm{KD} \rightarrow \longrightarrow \text { Actin }}$

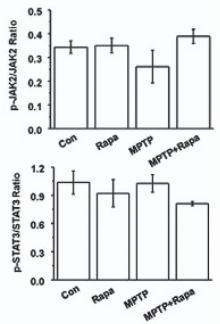

g
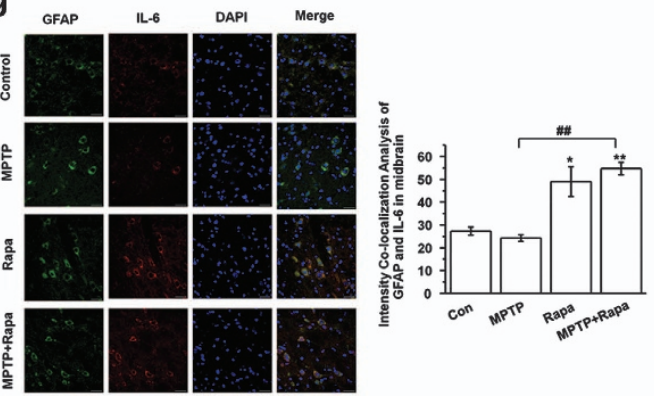

h
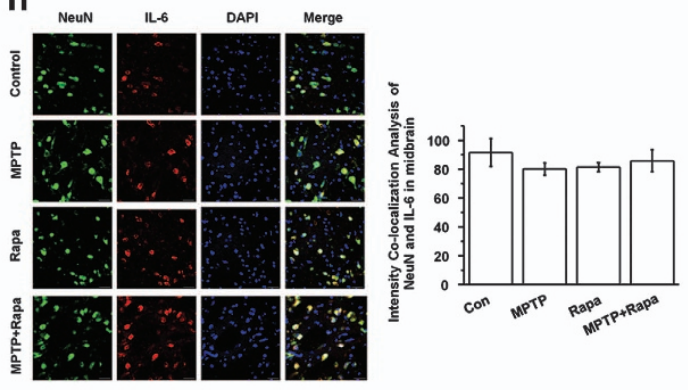

Figure 6 Rapamycin promotes IL-6 secretion in the serum and increases IL-6 expression in the midbrain of MPTP-treated mice. (a) ELISA of serum IL-6 levels ( $n=6$ per group, one-way analysis variance (ANOVA)). (b) IHC of IL-6 expression in the SNpc ( $n=6$ per group, one-way ANOVA). Scale bar, $40 \mu \mathrm{m}$. (c and d) qRT-PCR of IL-6 mRNA expression in the midbrain and striatum ( $n=6$ per group, one-way ANOVA). (e and f) Western blotting of JAK2/STAT3 expression in the midbrain and striatum ( $n=3$ per group, one-way ANOVA). ( $\mathbf{g}$ and $\mathbf{h}$ ) Immunofluorescence of IL-6 expression in astrocytes or neurons of the SNpc $(n=6$ per group, one-way ANOVA). Scale bar, $30 \mu \mathrm{m}$. Each bar represents the mean \pm S.E.M. of at least three independent experiments. ${ }^{*} P<0.05$, ${ }^{\star \star} P<0.01$, compared with the control group; ${ }^{\#} P<0.05$, ${ }^{\# \#} P<0.01$, for MPTP versus MPTP +Rapa group

+Rapa versus MPTP mice $(P<0.05$ and $P<0.01$, respectively) (Figure 6g), but rapamycin had no significant effect on neuronal IL-6 expression ( $P>0.05)$ (Figure 6h). These results verify the in vivo effects of rapamycin on IL-6 in MPTP-treated mice and support in vitro results suggesting that the effects occur at the level of astrocytes.

Rapamycin upregulates IL-6 and GLTs via the mTOR-Akt-NF-KB cascade. Because some effects on IL-6 expression were observed in the absence of regulation of the JAK2/STAT3 pathway, the regulation of additional cell signaling pathways was explored. Rapamycin decreased the in vitro and in vivo expression of phosphorylated S6, the downstream effector of mTORC1, which confirms that rapamycin engaged its target, mTORC1 $(P<0.01)$ (Figures $7 \mathrm{a}$ and $\mathrm{b}$, first 2 rows). In vitro Rapa+MPP ${ }^{+}$ treatment in astrocytes increased the expression of phosphorylated $\mathrm{PI3}$ kinase (PI3K), Akt, and NF-kB/p65 as compared with $\mathrm{MPP}^{+}$alone $(P<0.01$ and $P<0.05$, respectively) (Figure $7 \mathrm{a}$, last 10 rows), and in vivo MPTP+Rapa increased the expression of phosphorylated PI3K and NF- $\kappa \mathrm{B} /$ p65 compared with MPTP-only treatment $(P<0.05)$
(Figure $7 \mathrm{~b}$, last 10 rows), which suggests the possibility that these pathways may regulate $\mathrm{IL}-6$ expression. Therefore, to dissect the pathways regulating IL-6 expression, inhibitors of Akt (MK-2206), NF-kB (BAY 11-7082), and PI3K (LY 294002) were used. MK-2206 application did not significantly affect IL-6 expression in astrocytes treated with rapamycin+MPP ${ }^{+}$, $\mathrm{MPP}^{+}+$rapamycin, and $\mathrm{MPP}^{+}(P>0.05)$ (Figure $\left.7 \mathrm{c}\right)$, and only modestly reduced IL-6 secretion in astrocytes treated with rapamycin $\pm \mathrm{MPP}^{+}(P<0.01) \quad$ (Figure $\left.7 \mathrm{~d}\right)$. However, BAY 11-7082 robustly blocked the effects of rapamycin on IL-6 protein expression and secretion (Figure 7e, first row and Figure 7f), which suggests that NF- $K B$ is required for rapamycin function. BAY 11-7082 also robustly blocked the phosphorylation of the JAK2/STAT3 pathway (Figure 7e). Furthermore, MK-2206 and BAY 11-7082 eliminated the effects of rapamycin on GLTs expression and function in $\mathrm{MPP}^{+}$-treated astrocytes (Figures $8 \mathrm{a}-\mathrm{f}$ ), which suggests that Akt/NF- $k B$ pathway may also regulate the effects of rapamycin on GLT. LY 294002 also decreased IL-6 protein expression in $\mathrm{MPP}^{+}+$rapamycin- and $\mathrm{MPP}^{+}$-treated astrocytes groups (Supplementary Figure S2c), which suggests that PI3K may also be involved. These results support the possib- 
a

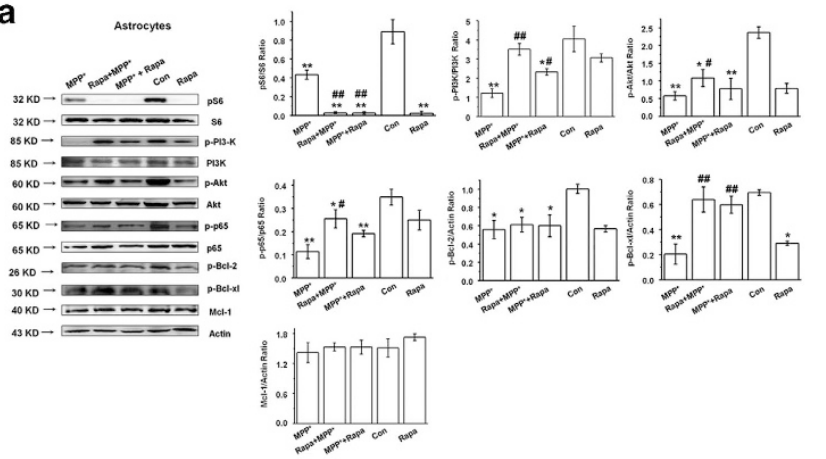

C
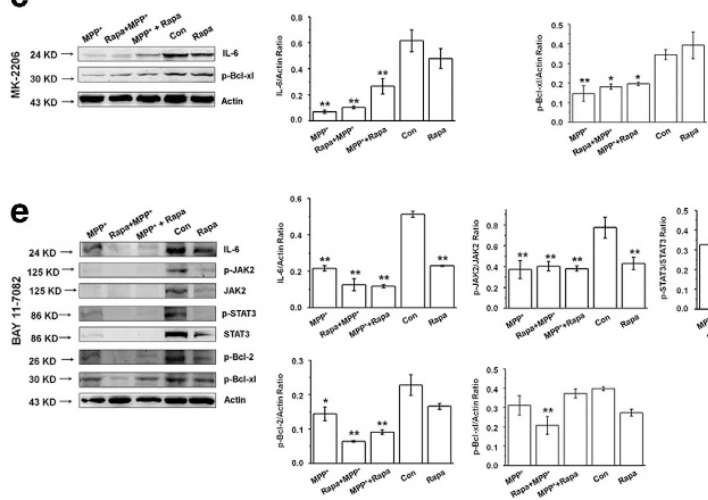

b

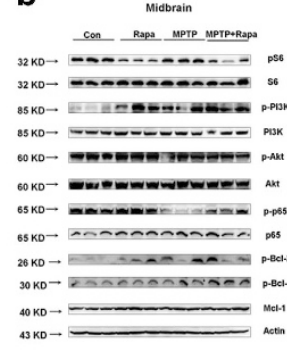

d

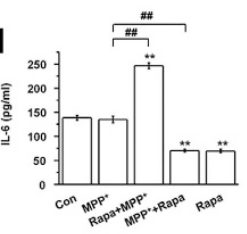

f

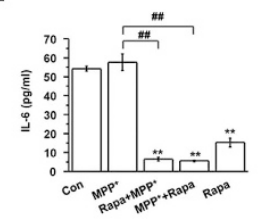

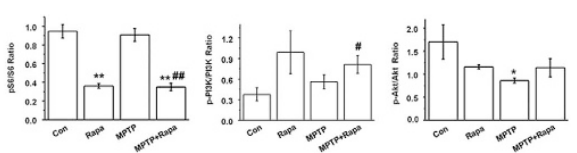
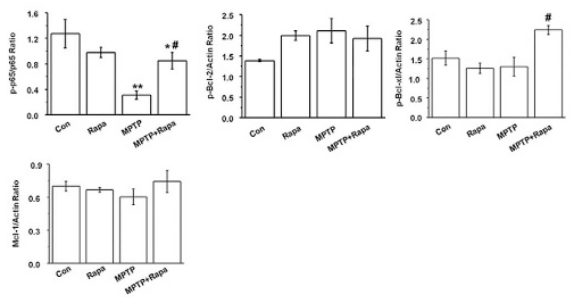

Figure 7 Rapamycin upregulates the IL-6/JAK2/STAT3/Bcl-xl pathway via the mTOR-Akt-NF- $\kappa B$ cascade. (a and $\mathbf{b}$ ) Western blotting of a panel of signal molecules in MPP+. treated astrocytes and in MPTP-treated mice (at least three experiments, one-way analysis of variance (ANOVA)). (c) The effect of MK-2206 on the expression of IL-6 and phospho (p)-Bcl-xl was determined by western blotting (at least three experiments, one-way ANOVA). (d) The effect of MK-2206 on IL-6 secretion in cell culture supernatant was determined by ELISA (at least three experiments in triplicate, one-way ANOVA). (e) The effect of BAY 11-7082 on the expression of IL-6/JAK2/STAT3, p-Bcl-2, and p-Bcl-xI was determined by western blotting (at least three experiments, one-way ANOVA). (f) The effect of BAY 11-7082 on IL-6 secretion in culture supernatants was determined by ELISA (at least three experiments in triplicate, one-way ANOVA). Each bar represents the mean \pm S.E.M. of at least three independent experiments. ${ }^{*} P<0.05,{ }^{* \star} P<0.01$, compared with the control group; and ${ }^{\#} P<0.05,{ }^{\#} P<0.01$, for $\mathrm{MPP}^{+}$versus Rapa+MPP${ }^{+}$or $\mathrm{MPP}^{+}+$Rapa

ility that activation of IL-6 by rapamycin in $\mathrm{MPP}^{+}$-treated astrocytes (and potentially in MPTP-treated mice) may be regulated predominantly by the mTOR-Akt-NF-KB cascade.

Rapamycin attenuates immune inflammation in MPTP-treated mice. The effects of rapamycin on other relevant immune cytokines were also tested. Interferon- $\gamma$ (IFN- $\gamma)$ signaling, together with tumor necrosis factor- $a$ (TNF- $a$ ), has an important role in stimulating and maintaining the activation of glial cells in PD. ${ }^{28,29}$ Also, IL-17 has been shown to induce IL-6 secretion, which further enhances Th17 cell differentiation. ${ }^{30}$ Transforming growth factor- $\beta 1$ (TGF- $\beta 1$ ) was proved to be a cofactor that potentiates the neurotrophic actions of glial cell line-derived neurotrophic factor (GDNF) in PD animal models. ${ }^{31}$ Besides, AAV2-mediated gene transfer of human IL-10 into the striatum shows neuroprotective role in MPTP model. ${ }^{32}$ Here we found that MPTP treatment led to an increase in serum IFN- $\gamma(P<0.01)$ and TNF- $a(P<0.05)$ (Figures $8 \mathrm{~g}$ and $\mathrm{h}$ ), but had no effect on IL-17 levels (Figure $8 \mathrm{i}, P>0.05$ ). MPTP also decreased TGF- $\beta 1$ and IL-10 mRNA expression in the midbrain $(0.61 \pm 0.01$ versus $1, P<0.05$ in Figure 8j; $0.47 \pm 0.14$ versus $1, P<0.05$ in Figure $8 \mathrm{k}$ ). Moreover, rapamycin significantly blocked the MPTP effects on serum IFN- $\gamma(P<0.01)$ and midbrain
TGF- $\beta 1$ mRNA $(1.04 \pm 0.04$ versus $0.61 \pm 0.01, P<0.05)$ (Figures $8 \mathrm{~g}$ and $\mathrm{j}$ ), but did not influence MPTP-induced changes in TNF- $a$ and IL-10 expression (Figures $8 \mathrm{~h}$ and $\mathrm{k}$, $P>0.05$ ). These findings indicate that MPTP has proinflammatory effects both in the periphery and in the CNS. A schematic summarizing these findings is shown in Figure 8 .

\section{Discussion}

Glial activation and inflammation are believed to contribute to PD and other aging-related diseases. ${ }^{33-37}$ Previously, three MPTP models are commonly used because of different MPTP dosing regimens. ${ }^{38-43}$ Within the subacute MPTP model, the dosing regimens refer to one injection of $20-30 \mathrm{mg} / \mathrm{kg}$ MPTP daily for five consecutive days. This model causes striatal dopamine depletion nearly $50 \%$ in C57BL/6 mice, and the DA lesion stabilizes by 21 days after MPTP administration. ${ }^{39,40}$ Besides, in this study, we focus on the expression changes of GLTs in PD and according to our previous work, we find the decreased GLTs, TH expression and movement disorder in different time points in the subacute MPTP model (unpublished data). Thus in this study we chose the subacute MPTP model. In the brain, MPTP is metabolized to 1-methyl-4phenyl-2,3-dihydropyridinium by the enzyme monoamine oxidase-B within non-DA cells, and then to MPP ${ }^{+}$. Generally, 

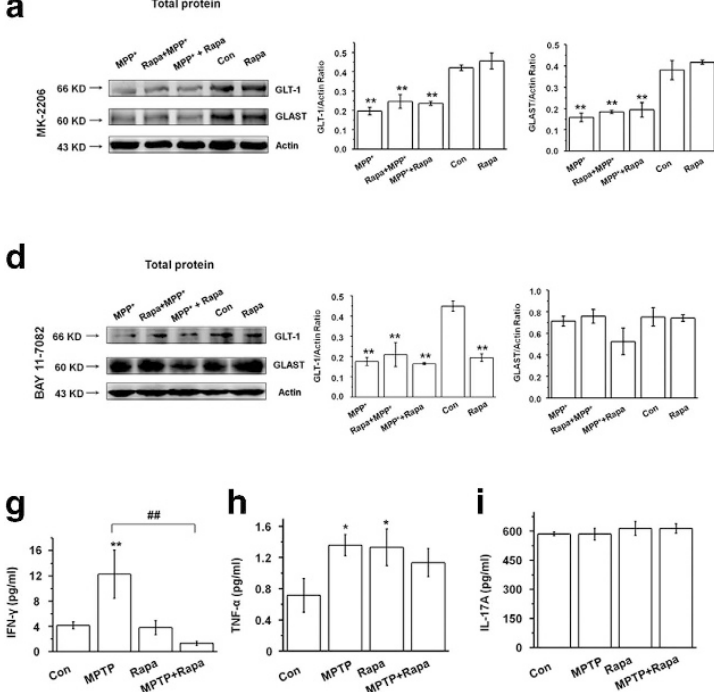

b

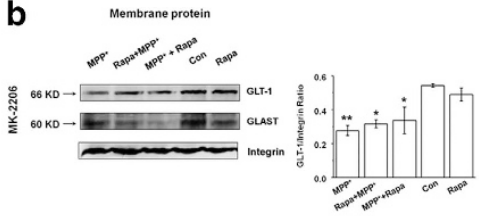

e
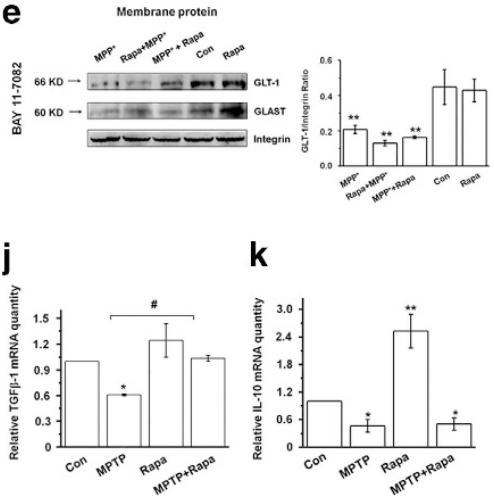

$\mathbf{k}$

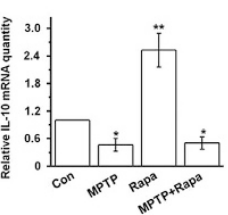

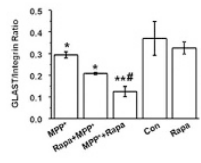

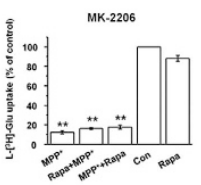

f
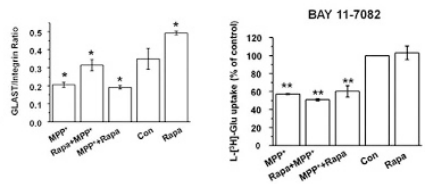

I

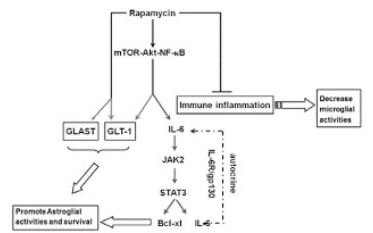

Figure 8 Rapamycin upregulates GLTs via the mTOR-Akt-NF- $\kappa$ B cascade in MPP ${ }^{+}$-treated astrocytes and attenuates immune inflammation in MPTP-treated mice. (a and $\mathbf{b}$ ) The effect of MK-2206 on the expression of GLT-1 and GLAST in total and membrane protein preparations was determined by western blotting (at least three experiments, one-way analysis of variance (ANOVA)). (c) The effect of MK-2206 on glutamate uptake in MPP ${ }^{+}$-treated astrocytes (at least three experiments in triplicate, one-way ANOVA). ( $\mathbf{d}$ and $\mathbf{e}$ ) The effect of BAY 11-7082 on GLT-1 and GLAST expression at the total and membrane level was determined by western blotting (at least three experiments, one-way ANOVA). (f) The effect of BAY 11-7082 on the glutamate uptake in MPP+-treated astrocytes (at least three experiments in triplicate, one-way ANOVA). Each bar represents the mean \pm S.E.M. of at least three independent experiments. ${ }^{*} P<0.05,{ }^{* *} P<0.01$, compared with the control group; and ${ }^{\sharp} P<0.05$, for MPP ${ }^{+}$versus MPP ${ }^{+}+$Rapa. $(\mathbf{g}-\mathbf{i})$ ELISA of IFN- $\gamma$, TNF- $\alpha$, and IL-17A levels in the serum of MPTP-treated mice ( $n=6$ per group, one-way ANOVA). ( $\mathbf{j}$ and $\mathbf{k}$ ) qRT-PCR of TGF- $\beta$ and IL-10 mRNA in MPTP-treated mice ( $n=6$ per group, one-way ANOVA). Each bar represents the mean \pm S.E.M. of at least three independent experiments. ${ }^{\star} P<0.05,{ }^{\star \star} P<0.01$, compared with the control group; ${ }^{\#} P<0.05$, ${ }^{\#} P<0.01$, for the MPTP versus MPTP+Rapa group. (I) Schematic model showing the neuroprotective mechanism of rapamycin in the PD models. Rapamycin upregulates GLTs via the mTOR-Akt-NF- $\kappa$ B cascade, in part, by decreasing the activity of Nedd4-2. Rapamycin also activates astroglial IL-6 signaling via the JAK2/STAT3/Bcl-xI pathway, although the expression of IL-6R is moderated via autocrine mechanisms. Through upregulation of GLTs and the IL-6/JAK2/STAT3 pathway, rapamycin promotes astroglial activities. In addition, rapamycin attenuates immune inflammation in PD models by decreasing microglial activities

the dose of $\mathrm{MPP}^{+}$used in primary mesencephalic DA neurons is slight, and a significant loss of primary DA neurons cultures is even detected at $0.5 \mu \mathrm{M} \mathrm{MPP}{ }^{+}{ }^{44,45}$ However, primary astrocytes are less susceptible to $\mathrm{MPP}^{+}$toxicity than DA neurons. According to a previous study and our work, astrocytes show significant loss at the dose of $800 \mu \mathrm{M}$ $\mathrm{MPP}^{+}{ }^{46,47}$ In this study, we also use the glial glutamate uptake results upon $\mathrm{MPP}^{+}$treatment as the indicator, and thus we chose $1000 \mu \mathrm{M}$ dose of $\mathrm{MPP}^{+}$as the work concentration.

Rapamycin has been shown to reduce MPTP toxicity; ${ }^{48}$ however, the mechanism has not been well characterized. Here we demonstrated that rapamycin has profound effects on the GLT expression in $\mathrm{MPP}^{+}$-treated astrocytes and MPTPtreated mice and prevents $\mathrm{MPP}^{+} / \mathrm{MPTP}^{-i n d u c e d}$ reduction in glutamate reuptake capacity. While the functional relevance of increased glutamate reuptake capacity has not been formally shown in this study, its relevance is well established in multiple models of PD. ${ }^{49-51}$ Therefore, it is reasonable to assume that the increase in glutamate reuptake capacity, at least in part, contributes to the neuroprotective effects of rapamycin in the MPTP model. It is well known that glutamate-mediated transmission has a central role in numerous fundamental brain functions, such as synaptic plasticity. Our study is the first to show that rapamycin prevents MPTP-induced hippocampal LTP impairment. This result further supports previous demonstration of cognitive dysfunction in the early phase of
MPTP model. ${ }^{52}$ Also, GLT-1 has been demonstrated to be responsible for the increase in glutamate uptake during LTP, ${ }^{53}$ and we found that rapamycin can restore the GLT-1 expression in the hippocampus. Thus, upregulation of GLT-1 upon rapamycin treatment may improve the LTP in MPTP mouse lesions.

We also found that rapamycin reduced the number of astrocytes staining double positive for GLT-1+ubiquitin, GLAST+ubiquitin, GLT-1+phospho-Nedd4-2, and GLAST +phospho-Nedd4-2 in MPTP-treated mice. Consistent with these findings, regulation of GLT via Nedd4-2 has been reported in alternative neurodegenerative disease models. ${ }^{54-56}$ Our recent unpublished data also suggest that Nedd4-2 knockdown ameliorates movement disorders and increases TH expression in PD mice via decreasing GLT ubiquitination. Thus, in this study we offer a possible role of rapamycin in regulating GLT levels by inhibiting Nedd4-2dependent ubiquitination in the PD model.

In addition, rapamycin also increased the concentration of IL- 6 in the midbrain of MPTP-treated mice and these results were recapitulated in MPP ${ }^{+}$-treated astrocytes. IL- 6 can have both proinflammatory and anti-inflammatory effects, and its role in PD remains controversial. ${ }^{18-22,57,58}$ However, MPTP treatment upregulated the proinflammatory markers IFN- $\gamma$ and TNF- $a$ and downregulation of the anti-inflammatory markers TGF- $\beta 1$ and IL-10 in the periphery and the CNS, which 
suggests that MPTP induces a proinflammatory profile. Rapamycin partially reversed the MPTP-induced changes in immune markers, which indicates that the rapamycinmediated increase in IL-6 levels may be predominantly anti-inflammatory.

We further used pharmacological inhibitors to investigate the cell signaling cascades regulated by rapamycin. Inhibition of NF- $K \mathrm{~B}$ led to the most robust blockade of rapamycinmediated regulation of GLT, IL-6, and JAK2/STAT3 phosphorylation. Therefore, activation of NF- $\kappa \mathrm{B}$ by rapamycin appears to be a step fairly proximal in the cell signaling cascade that is crucial for mediating the biological effects of rapamycin, whereas PI3K/Akt signaling cascade may be partially responsible for mediating the effects of rapamycin. The findings in this study are also supported by other reports. ${ }^{59,60}$

Previously, the IL-6/JAK/STAT pathway have been proved to regulate $\mathrm{GLTs},{ }^{61,62}$ so actually we also want to explore whether the IL-6/JAK/STAT pathway is involved in regulating GLTs in PD. However, here we did not find the connection between the JAK/STAT pathway and GLTs. In our hand, we found that rapamycin increased GLT expression in SNpc (Figure 2c), whereas the JAK/STAT pathway show no obvious change in SNpc (Figure 6e); besides, both pre- and posttreatment of rapamycin increased GLT-1 expression in $\mathrm{MPP}^{+}$treated astrocytes (Figures $2 \mathrm{f}$ and $\mathrm{g}$ ); however, the JAK/STAT pathway only showed significant changes in pre-treatment of rapamycin (Figure 5a). These results suggest that JAK/STAT pathway may share little connection with GLTs in PD, thus we choose to state the neuroprotective effects of IL-6/JAK/STAT pathway in another story. Intriguingly, both GLTs and IL-6 are regulated by the $m$ TOR-Akt-NF- $\kappa$ B cascade, suggesting these two stories are linked to some extent.

In summary, the neuroprotective effects of rapamycin in the MPTP model have for the first time been associated with direct effects of rapamycin on astrocytes that lead to improved glutamate reuptake capacity and, in the presence of elevated IL-6 levels, to a less inflammatory cytokine profile. These findings expand our knowledge about the protective mechanisms of rapamycin in an animal model of $P D$ and provide additional targets for therapeutic interventions in PD.

\footnotetext{
Materials and Methods

Reagents. MPP ${ }^{+}$and MPTP were purchased from Sigma-Aldrich (St. Louis, MO, USA). Dulbecco's modified Eagle's medium/F12 and fetal calf serum were purchased from Hyclone (Logan, UT, USA). Anti-TH and GLT-1 antibodies were purchased from Millipore (Bedford, MA, USA). Anti-GLAST, anti- $\alpha$-synuclein, antiIL-6, and anti-IL-6 receptor antibodies were purchased from Santa Cruz Biotechnology (Santa Cruz, CA, USA). Anti-phospho-Nedd4-2 (Ser342), antiNedd4-2, anti-phospho-S6, and anti-S6 antibodies were purchased from Cell Signaling Technology (Danvers, MA, USA). Anti-phospho-JAK2 (Tyr221), antiphospho-NF- $\kappa B$ p65 (Ser276), anti-phospho-STAT3 (Tyr705), anti-JAK2, anti-NF- $\kappa B$ p65, anti-STAT3, anti-gp130, anti-phospho-PI3K p85- $\alpha$ (Tyr607), anti-PI3K p85- $\alpha$, anti-phospho-Akt (Ser473), anti-Akt, anti-phospho-Bcl-2 (Ser87), and anti-phosphoBcl-xl (Ser62) antibodies were purchased from EnoGene (Nanjing, China). Rapamycin was purchased from Sangon (Shanghai, China). Actin antibody was purchased from Beyotime (Shanghai, China). PI3K inhibitor (LY 294002), Akt inhibitor (MK-2206), and NF- $k B$ (BAY 11-7082) were purchased from Selleck Chemicals (Houston, TX, USA). Anti-Mcl-1, FITC-conjugated goat anti-mouse, TRITC-conjugated goat anti-rabbit, horseradish peroxidase (HRP)-conjugated goat anti-mouse, and rabbit antibodies were purchased from Boster (Wuhan, China). EZLink Sulfo-NHS-SS-Biotin was purchased from Thermo Scientific (Waltham, MA, USA; no. 21331). Trizol was purchased from Invitrogen (Carlsbad, CA, USA).
}

PrimeScript RT Reagent Kits and SYBR Premix Ex Taq Kits were purchased from Takara (Otsu, Japan). IL-6, TNF- $\alpha$, IL-17A, and IFN- $\gamma$ Enzyme-linked Immunosorbent Assay (ELISA) Kits were purchased from BioLegend (San Diego, CA, USA). TUNEL (terminal deoxynucleotidyl transferase-mediated dUTP nick-end labeling) Staining Assay Kits were purchased from Beyotime.

Animals and cell culture. Ten-week-old, C57BL/6 male mice were obtained from the Guangdong Medical Laboratory Animal Facility (Foshan, China). Primary cortical astrocytes were obtained from newborn C57BL/6 mice as described. ${ }^{46}$ Primary astrocytes, BV2 cells, and PC12 cells were cultured in Dulbecco's modified Eagle's medium/F12 supplemented with $10 \%$ fetal calf serum. All animal protocols in this study were approved by the Institutional Animal Care and Use Committee of the Southern Medical University.

Drug treatment. Stock solutions of $\mathrm{MPP}^{+}$were prepared in $0.01 \mathrm{M}$ PBS and rapamycin were prepared in DMSO. Stock solutions were then diluted in cell culture medium containing half-serum to the appropriate concentrations. The addition of $\mathrm{MPP}^{+}$to astroglial cultures was performed at $1000 \mu \mathrm{M}$ as stated by our work and others, ${ }^{46,63}$ and rapamycin was performed at $100 \mathrm{nM}$ as stated by the previous study. ${ }^{59}$ For in vitro assays, cells were randomly divided into five groups: (1) control group: cells were treated with normal culture medium for $24 \mathrm{~h}$; (2) MPP ${ }^{+}$group: cells were treated with culture medium containing $1000 \mu \mathrm{M} \mathrm{MPP}^{+}$for $24 \mathrm{~h}$; (3) Rapa $+\mathrm{MPP}^{+}$group: cells were pretreated with culture medium containing $100 \mathrm{nM}$ rapamycin for $4 \mathrm{~h}$, rapamycin was removed, and cells were treated with culture medium containing $1000 \mu \mathrm{M} \mathrm{MPP}{ }^{+}$for $24 \mathrm{~h}$; (4) $\mathrm{MPP}^{+}+$Rapa group: cells were treated with culture medium containing $1000 \mu \mathrm{M} \mathrm{MPP}+$ for $24 \mathrm{~h}, \mathrm{MPP}^{+}$was removed, and cells were treated with culture medium containing $100 \mathrm{nM}$ rapamycin for 4 h; (5) Rapa group: cells were treated with culture medium containing $100 \mathrm{nM}$ rapamycin for $4 \mathrm{~h}$, rapamycin was removed, and cells were treated with normal culture medium for $24 \mathrm{~h}$. The control group was treated with PBS or DMSO.

For in vivo experiments, mice were randomly divided into four groups: saline +vehicle (Con), MPTP+vehicle (MPTP), saline+rapamycin (Rapa), and MPTP +rapamycin (MPTP+Rapa). According to the previous studies, ${ }^{38,64}$ MPTP was administered intraperitoneally for 5 consecutive days at a dose of $30 \mathrm{mg} / \mathrm{kg}$ free base (MPTP-HCl) in saline. The vehicle for rapamycin was DMSO. Rapamycin or vehicle was administered for 11 consecutive days, starting 2 days before MPTP treatment, at a dose of $7.5 \mathrm{mg} / \mathrm{kg}$, as described. ${ }^{13}$ MPTP/saline injections were performed at 0800 hours and rapamycin/vehicle injections were performed at 2000 hours. One day after the last rapamycin/vehicle injections, behavioral experiments were performed and the animals were killed for tissue collection.

Behavioral tests. Grasping test. Mice were tested with the grasping experiment at different time points, respectively. Briefly, mice were suspended by their forelimbs on a metal rod (diameter: $1.5 \mathrm{~mm}$ ) and positioned $\sim 30 \mathrm{~cm}$ above the box. The holding time on the metal rod was recorded.

Pole-climbing test. Mice were placed on the peak of foam ball (diameter: $2.0 \mathrm{~cm}$ ) fixed on the stick (diameter: $1.0 \mathrm{~cm}$; length: $50 \mathrm{~cm}$ ). The climb time from the peak to the bottom of the stick was counted

IHC assay. Brain tissue samples were embedded in optimum cutting temperature compound (Sakura Finetek, Torrance, CA, USA) and stored at $-80^{\circ} \mathrm{C}$. Samples sections were cut into $10 \mu \mathrm{m}$ slices, antigen retrieval was performed using citrate buffer, and endogenous peroxidase activity was blocked with $3 \% \mathrm{H}_{2} \mathrm{O}_{2}$ for $10 \mathrm{~min}$ at room temperature (RT). Samples were incubated with the indicated primary antibodies overnight at $4{ }^{\circ} \mathrm{C}$. After washing with PBS, slices were incubated with HRP-conjugated secondary antibodies (ZSGB-BIO, Beijing, China) for $1 \mathrm{~h}$ at RT. Antibody-peroxidase complexes were revealed by incubating slices with a 3,3-Diaminobenzidine Peroxidase Substrate Kit (Boster, Wuhan, China). The integrated option density was counted at $\times 400$ magnification in at least 10 highpower fields from kidney sections from representative mice in each group using an Image-Pro Plus 6.0 photogram analysis system (IPP 6.0; Media Cybernetics, Bethesda, MD, USA).

Immunofluorescence. Brain tissue slices were obtained as described for IHC. Slices were fixed in $4 \%$ paraformaldehyde, rinsed with PBS, permeabilized with $0.1 \%$ Triton X-100, and blocked with $1 \%$ BSA. Slices were incubated with primary antibodies overnight at $4{ }^{\circ} \mathrm{C}$, rinsed with PBS, and incubated with FITCconjugated goat anti-mouse IgG or TRITC-conjugated goat anti-rabbit lgG for $2 \mathrm{~h}$ at $37^{\circ} \mathrm{C}$. DAPI was used to stain cell nuclei. Immunostaining was then examined using 
the Olympus $1 \times 81$ FV1000 Laser Scanning Confocal Microscope (Shinjuku, Tokyo, Japan). Primary antibodies were replaced with $1 \%$ BSA as a negative control.

Colocalized immunofluorescence assay. Brain tissue slices were fixed in $4 \%$ paraformaldehyde and then rinsed with PBS. Then, the slices were permeabilized with $0.1 \%$ Triton X-100 and blocked with $5 \%$ BSA. For the colocalization study, the slices were incubated with primary antibodies (as indicated in the Figures $3 a, b, 4 b, c$, and $6 g$ and $h$ ) overnight at $4{ }^{\circ} \mathrm{C}$, rinsed with PBS and incubated with FITC-conjugated goat anti-mouse IgG and TRITC-conjugated goat anti-rabbit lgG for $2 \mathrm{~h}$ at $37^{\circ} \mathrm{C}$. DAPI was used to stain cell nuclei. Immunostaining was then examined using an Olympus $1 \times 81$ FV1000 Laser Scanning Confocal Microscope (Shinjuku, Tokyo, Japan). Quantification of double-labeled immunocytological antigens that were colocalized was performed by imaging and analyzing cells using IPP 6.0 (Media Cybernetics, Bethesda, MD, USA). Generally, GLT-1, GLAST, or IL-6 clusters were selected automatically in the pseudocolored 'red' channel as discrete puncta of intensity $>1.5$-fold brighter than the background fluorescence. Selected clusters were transferred to the green channel to measure the Ub, phospho-Nedd4-2, GFAP, or NeuN fluorescence. Colocalization of these targeted proteins was measured as the percentage of integrated Ub, phosphoNedd4-2, GFAP, or NeuN pixel intensities that overlapped with the GLT-1, GLAST, or IL-6 fluorescence in individual clusters. As a negative control, the primary antibody was replaced with $5 \%$ BSA.

TUNEL staining. Apoptotic cells in the SNpc were stained by TUNEL assay according to the manufacturer's instructions (Beyotime). Briefly, brain tissue slices were obtained as described for IHC, and the slices in each group were fixed in $4 \%$ paraformaldehyde and rinsed with PBS. Then, the slices were permeabilized with $1.0 \%$ Triton X-100 for $5 \mathrm{~min}$ and blocked with $5 \%$ BSA for $30 \mathrm{~min}$. The fluorescein TUNEL reagent mixture was applied for $60 \mathrm{~min}$ at $37^{\circ} \mathrm{C}$. DAPI was used to stain cell nuclei, and then the slices were viewed under a laser scanning confocal microscope.

qRT-PCR. Total RNA was isolated from brain tissues using Trizol Reagent (Life Technologies, Carlsbad, CA, USA) and cDNA was synthesized using a TaKaRa PrimeScript Reagent Kit with the gDNA Eraser (TaKaRa, Otsu, Japan). The cDNA was then amplified with SYBR Premix Ex Taq (TaKaRa, Otsu, Japan) according to the manufacturer's instructions. Primers used in this study are listed as follows: GLT-1 (forward) 5'-CGATGAGCCAAAGCACCGAA-3' and (reverse) 5'-CTGGAGAT GATAAGAGGGAGGATG-3'; GLAST (forward) 5'-TCAAGTTCTGCCACCCTACC-3' and (reverse) 5'-TCTGTCCAAAGTTCAGGTCAA-3'; IL-6 (forward) 5'-ACTTCCATC CAGTTGCCTTC-3' and (reverse) 5'-ATTTCCACGATTTCCCAGAG-3'; TGF- $\beta 1$ (forward) and 5'-ATTCCTGGCGTTACCTTGG-3' (reverse) 5'-AGCCCTGTAT TCCGTCTCCT-3'; IL-10 (forward) 5'-GCAGCCTTGCAGAAAAGAGAG-3' and (reverse) TCCTGCATTAAGGAGTCGGTT; $\beta$-actin (forward) 5 '-CTACAATGAGCT GCGTGTGGC-3' and (reverse) CAGGTCCAGACGCAGGATGGC. Fluorescence was detected using a Corbett Research RG-6000 Real-Time PCR Machine (Corbett Life Science, Sydney, NSW, Australia). Each sample was run in triplicate and was compared with actin as the internal control. Results were obtained by using the $2^{-\Delta \Delta C T}$ method. Briefly, the $\mathrm{Ct}$ values of the gene of interest and actin were derived from the software in the real-time PCR machine. $\Delta \mathrm{Ct}=\mathrm{Ct}$ (gene of interest) $-\mathrm{Ct}($ actin) $; \Delta \Delta \mathrm{Ct}=((\mathrm{Ct}$ gene of interest $-\mathrm{Ct}$ actin) rapamycin, $\mathrm{PD}$ or rapamycin+PD) - ((Ct gene of interest $-\mathrm{Ct}$ actin) control); and finally, the $2^{-\Delta \Delta C T}$ value represents the relative expression of the gene of interest in rapamycin, $\mathrm{PD}$, or rapamycin+PD groups compared with control. Data are from three separate experiments, and each was performed in triplicate.

Total protein extraction. For the total membrane protein extraction, cells or brain tissues were lysed with radioimmunoprecipitation assay buffer (Beyotime) containing $1 \mathrm{mM}$ PMSF (Beyotime). The protein concentration was measured via BCA Assay Kit (Beyotime, Shanghai, China). Samples were diluted in protein loading buffer, and heated to $95^{\circ} \mathrm{C}$ for $5 \mathrm{~min}$.

Cell surface biotinylation. Cell surface expression levels of GLT-1 and GLAST were tested using the membrane-impermeable biotinylation reagent EZ-Link Sulfo-NHS-SS-Biotin. Cells or tissues were washed two times with ice-cold PBS (pH 8.0) and then incubated with $2.5 \mathrm{ml}$ of EZ-Link Sulfo-NHS-SS-Biotin $(0.5 \mathrm{mg} / \mathrm{ml}$ in PBS) in two successive 20 min incubations on ice with gentle shaking. Cells or tissues were washed two times with $100 \mathrm{mM}$ glycine to remove non-reacted biotinylation reagent by incubation on ice for $20 \mathrm{~min}$. Then, the cells or homogenized tissues were lysed on ice for $20 \mathrm{~min}$ in $750 \mu \mathrm{l}$ of cell lysis buffer containing the protease inhibitor mixture and $1 \mathrm{mM}$ PMSF. The debris was removed by centrifugation at $12000 \times \mathrm{g}$ for $20 \mathrm{~min}$ at $4^{\circ} \mathrm{C}$. Supernatants were transferred to new tubes and $200 \mu \mathrm{l}$ of streptavidin-agarose beads were added to bind the biotinlabeled cell membrane proteins. They were centrifuged at $3000 \times \mathrm{g}$ for $1 \mathrm{~min}$ at $4{ }^{\circ}$ $\mathrm{C}$ and the supernatant was discard, and then were washed three times with ice-cold lysis buffer. At the end, they were washed once with ice-cold PBS and centrifuged at $16000 \times g$ for 1 min at $4{ }^{\circ} \mathrm{C}$. The membrane protein samples were used for western blotting.

Western blotting assay. Membrane and total protein samples were resolved via $12 \%$ SDS-PAGE and transferred to polyvinylidene difluoride (PVDF) membranes. After blocking with $5 \% \mathrm{BSA}$ for $2 \mathrm{~h}$ at RT, the PVDF membranes were incubated with the indicated primary antibodies overnight at $4{ }^{\circ} \mathrm{C}$. The PVDF membranes were incubated with HRP-conjugated secondary antibodies followed by three washes with TBS-T (Tris-buffered saline containing $0.1 \%$ Tween-20). Proteins were visualized with enhanced chemiluminescence (Beyotime). Actin immunoreactivity was set as loading control for each protein of interest.

L- $\left[{ }^{3} \mathrm{H}\right]$-Glutamic acid uptake assay. L- $\left[{ }^{3} \mathrm{H}\right]$-Glutamic acid uptake assays were performed as described previously. ${ }^{46}$ Briefly, astrocytes were washed once with choline solution (150 mM choline chloride, $5 \mathrm{mM} \mathrm{KP}, \mathrm{pH} 7.4,0.5 \mathrm{mM} \mathrm{MgSO}_{4}$, and $0.3 \mathrm{mM} \mathrm{CaCl}$ ), and then $0.4 \mu \mathrm{Ci}$ per well $\mathrm{L}-\left[{ }^{3} \mathrm{H}\right]$-glutamic acid (specific activity $12.9 \mathrm{Ci} / \mathrm{mmol}$ ) were added to the wells. The reactions were incubated for $10 \mathrm{~min}$ at $\mathrm{RT}$ and stopped by two additions of ice-cold $\mathrm{NaCl}$ solution $(150 \mathrm{mM} \mathrm{NaCl}, 5 \mathrm{mM}$ $\mathrm{KP}_{\mathrm{i}}, \mathrm{pH} 7.4,0.5 \mathrm{mM} \mathrm{MgSO}_{4}$, and $0.3 \mathrm{mM} \mathrm{CaCl}_{2}$ ). One percent SDS was added to dissolve cells, and radioactivity was measured by liquid scintillation counting. Data are from three separate experiments, each performed in triplicate.

Determination of glutamate uptake in the synaptosomes. Midbrain and striatum tissues were homogenized in $0.32 \mathrm{M}$ sucrose solution $(0.32 \mathrm{M}$ sucrose, $5 \mathrm{mM}$ HEPES, $\mathrm{pH} \mathrm{7.4)}$ and centrifuged at $1000 \times \mathrm{g}$ for $15 \mathrm{~min}$. The resulting supernatants were centrifuged further at $15000 \times \mathrm{g}$ for $30 \mathrm{~min}$, and the pellets were resuspended using $0.32 \mathrm{M}$ sucrose solution to obtain the crude synaptosomes. Protein concentrations were determined by BCA Colorimetric Assay (Beyotime, Shanghai, China). Then, the crude synaptosomes were resuspended in Kreb's buffer (127 mM NaCl, $3.73 \mathrm{mM} \mathrm{KCl}, 1.8 \mathrm{mM} \mathrm{CaCl}_{2}, 1.18 \mathrm{mM} \mathrm{KH}_{2} \mathrm{PO}_{4}$, $20 \mathrm{mM} \mathrm{NaHCO} 3,2 \mathrm{mM}$ ATP, $2 \mathrm{~g} / \mathrm{l}$ D-glucose, pH 7.4) in a water bath at $25^{\circ} \mathrm{C}$ for $10 \mathrm{~min}$ at a final concentration of $0.5 \mathrm{mg} / \mathrm{ml}$. L- $\left[{ }^{3} \mathrm{H}\right]$-glutamic acid of $1 \mu \mathrm{Ci}$ was added to the synaptosome preparations in each tube in a water bath at $25^{\circ} \mathrm{C}$ for another $10 \mathrm{~min}$, and the reactions were terminated with $5 \mathrm{ml}$ of ice-cold Kreb's buffer. Synaptosomes were washed three times to remove excess labeled glutamate with phosphate-buffered saline and filtrated with $0.22 \mu \mathrm{m}$ filter paper. The filter paper containing the rinsed synaptosomes were then transferred into scintillation vials containing $3 \mathrm{ml}$ of scintillation cocktail in the dark overnight. The radioactivity was measured by liquid scintillation counting on the next day. The glutamate uptake in the synaptosomes was represented in c.p.m. per mg protein per min. Data are from three separate experiments, each performed in triplicate.

Enzyme-linked immunosorbent assay (ELISA). Cytokines in the serum and culture supernatants were measured with ELISA MAX Deluxe Sets (BioLegend) according to the kit instructions. Briefly, each plate was coated with $100 \mu \mathrm{l}$ of the capture antibody solution and incubated overnight at $4^{\circ} \mathrm{C}$. Plates were washed four times with wash buffer, and $1 \mathrm{x}$ assay diluent solution was added to block nonspecific binding. After incubation for $1 \mathrm{~h}$ at RT, sera were added to the wells and incubated for $2 \mathrm{~h}$ at RT. Then, plates were incubated with indicated antibodies for $1 \mathrm{~h}$ at RT. After four washes, plates were incubated with diluted avidinHRP solutions for $30 \mathrm{~min}$ at RT. After five washes, mixed tetramethylbenzidine substrate was added to each well and the absorbance at $450 \mathrm{~nm}$ was measured with a microplate reader (Perkin-Elmer, Waltham, MA, USA). Data were obtained from three separate experiments, each performed in triplicate.

Hippocampal slice preparations. Mice (C57BL/6 $\times 129)$ were killed by isoflurane anesthesia at age 6-8 weeks for field recordings. Brains were quickly removed and submerged in ice-cold oxygenated sucrose-replaced artificial cerebrospinal fluid (ACSF) cutting solution containing (in $\mathrm{mM}$ ): 206 sucrose, 2 $\mathrm{KCl}, 2 \mathrm{MgSO}_{4}, 1.25 \mathrm{NaH}_{2} \mathrm{PO}_{4}, 1 \mathrm{CaCl}_{2}, 1 \mathrm{MgCl}_{2}, 26 \mathrm{NaHCO}_{3}, 10$ d-glucose, $\mathrm{pH}$ $7.4,315 \mathrm{mOsm}$. Transverse slices (350 $\mu \mathrm{m}$ thick) were cut with a vibroslicer from the middle portion of each hippocampus. After dissection, slices were incubated in 
ACSF containing (in mM): $124 \mathrm{NaCl}, 2 \mathrm{KCl}, 2 \mathrm{MgSO}_{4}, 1.25 \mathrm{NaH}_{2} \mathrm{PO}_{4}, 2.5 \mathrm{CaCl}_{2}, 26$ $\mathrm{NaHCO}_{3}, 10$ D-glucose, pH 7.4, $310 \mathrm{mOsm}$, in which they were allowed to recover for at least $90 \mathrm{~min}$ before recording. A single slice was then transferred to the recording chamber and submerged beneath continuously perfused ACSF saturated with $95 \% \mathrm{O}_{2}$ and $5 \% \mathrm{CO}_{2}$. Slices were incubated in this chamber for $20 \mathrm{~min}$ before stimulation at RT $\left(\sim 24{ }^{\circ} \mathrm{C}\right)$.

Electrophysiology. Standard field excitatory postsynaptic potentials (fEPSPS) were recorded in the CA1 region of hippocampus. A bipolar-stimulating electrode (FHC Inc., Bowdoin, ME, USA) was placed in the Schaffer collaterals to deliver test and conditioning stimuli. A borosilicate glass recording electrode filled with ACSF was positioned in the stratum radiatum of CA1, 200-300 $\mu \mathrm{m}$ from the stimulating electrode. fEPSPs in CA1 were induced by test stimuli at $0.05 \mathrm{~Hz}$ with an intensity that elicited a fEPSP amplitude of $40-50 \%$ of maximum. Test responses were recorded for 30-60 min before beginning the experiments to ensure stability of the response. To induce LTP, two consecutive trains (1 s) of stimuli at $100 \mathrm{~Hz}$ separated by $20 \mathrm{~s}$. A protocol that induces LTP lasting $\sim 1.5 \mathrm{~h}$ in wild-type mice of this genetic background were applied to the slices. The field potentials were amplified $100 \times$ using Axon Instruments 700B amplifier (Foster City, CA, USA) and digitized with Digidata 1322A (Foster City, CA, USA). The data were sampled at $10 \mathrm{kHz}$ and filtered at $2 \mathrm{kHz}$. Traces were obtained by pClamp 9.2 and analyzed using the Clampfit 9.2 (Molecular Devices, CA, USA).

Statistical analysis. Statistical analysis of data was performed by the Dunnett's or LSD post-test based on the ANOVA for multivariate data analysis using SPSS 16.0 (SPSS Inc., Chicago, IL, USA). Data are expressed as means \pm S.E. of three independent experiments. Results were considered statistically significant at ${ }^{*} P<0.05$ (and ${ }^{*} P<0.01$ ).

\section{Conflict of Interest}

The authors declare no conflict of interest.

Acknowledgements. This work was supported by grants from the Nationa Natural Science Foundation of China (Grant Nos 31170734, 31570716, U1603281 to SQ and 81271428, 81471292, U1503222, 81430021 to PX), the Science and Technology Planning Project of Guangdong Province (Grant Nos 2012B050200003, 2013B021800305, 2016A050502025 to SQ), the Science and Technology Planning Project of Guangzhou (Grant No. 2013J4500018 to SQ and 2014J4100008 to XZ), the Scientific and Technological Innovation Programs of Higher Education Institution in Guangdong (Grant No. 2013KJCX0041 to SQ), and the Alzheimer's Association (NIRG-12-242825 to SL).

\section{Author contributions}

$S Q, P X$ and $Y Z$ conceived and designed the molecular biology experiments in this study. SL designed the electrophysiology experiments. ML and ZW performed the electrophysiology experiments. YZ performed the Western blotting and $L-\left[{ }^{3} \mathrm{H}\right]$ glutamic acid uptake assays. XZ and LW performed behavioral tests. XH carried out immunohistochemistry, immunocytochemistry and ELISA assays. XW performed the qRT-PCR and determination of glutamate uptake in the synaptosomes. $Y Z, X H, X W$, $S L$ and $S Q$ analyzed the data. YZ, SL and $S Q$ wrote the manuscript.

1. Michel PP, Hirsch EC, Hunot S. Understanding dopaminergic cell death pathways in Parkinson disease. Neuron 2016; 90: 675-691.

2. McCann $\mathrm{H}$, Cartwright $\mathrm{H}$, Halliday GM. Neuropathology of $\alpha$-synuclein propagation and braak hypothesis. Mov Disord 2016; 31: 152-160.

3. Banerjee R, Starkov AA, Beal MF, Thomas B. Mitochondrial dysfunction in the limelight of Parkinson's disease pathogenesis. Biochim Biophys Acta 2009; 1792: 651-663.

4. Blesa J, Trigo-Damas I, Quiroga-Varela A, Jackson-Lewis VR. Oxidative stress and Parkinson's disease. Front Neuroanat 2015; 9: 91.

5. Ambrosi G, Cerri S, Blandini F. A further update on the role of excitotoxicity in the pathogenesis of Parkinson's disease. J Neural Transm 2014; 121: 849-859.

6. Blandini F. An update on the potential role of excitotoxicity in the pathogenesis of Parkinson's disease. Funct Neurol 2010; 25: 65-71.

7. Gardoni F, Di Luca M. Targeting glutamatergic synapses in Parkinson's disease. Curr Opin Pharmacol 2015; 20: 24-28.

8. Fakhoury M. Role of immunity and inflammation in the pathophysiology of neurodegenerative diseases. Neurodegener Dis 2015; 15: 63-69.
9. Macchi B, Di Paola R, Marino-Merlo F, Felice MR, Cuzzocrea S, Mastino A. Inflammatory and cell death pathways in brain and peripheral blood in Parkinson's disease. CNS Neurol Disord Drug Targets 2015; 14: 313-324.

10. Beal MF. Excitotoxicity and nitric oxide in Parkinson's disease pathogenesis. Ann Neurol 1998; 44: S110-S114.

11. Wang H, Shimoji M, Yu SW, Dawson TM, Dawson VL. Apoptosis inducing factor and PARP. mediated injury in the MPTP mouse model of Parkinson's disease. Ann NYAcad Sci 2009; 991: $132-139$.

12. Johnson SC, Rabinovitch PS, Kaeberlein M. mTOR is a key modulator of ageing and age-related disease. Nature 2013; 493: 338-345.

13. Malagelada C, Jin ZH, Jackson-Lewis V, Przedborski S, Greene LA. Rapamycin protects against neuron death in in vitro and in vivo models of Parkinson's disease. J Neurosci 2010; 30: $1166-1175$.

14. Li S, Hong S, Shepardson NE, Walsh DM, Shankar GM, Selkoe D. Soluble oligomers of amyloid beta protein facilitate hippocampal long-term depression by disrupting neuronal glutamate uptake. Neuron 2009; 62: 788-801.

15. Lei M, Xu H, Li Z, Wang Z, O'Malley TT, Zhang D et al. Soluble A $\beta$ oligomers impair hippocampal LTP by disrupting glutamatergic/GABAergic balance. Neurobiol Dis 2016; 85: 111-121.

16. Zhu G, Huang $\mathrm{Y}, \mathrm{Chen} \mathrm{Y}$, Zhuang $\mathrm{Y}$, Behnisch T. MPTP modulates hippocampal synaptic transmission and activity-dependent synaptic plasticity via dopamine receptors. J Neurochem 2012; 122: 582-593.

17. Sidoryk-Wegrzynowicz M, Lee ES, Ni M, Aschner M. Manganese-induced downregulation of astroglial glutamine transporter SNAT3 involves ubiquitin-mediated proteolytic system. Glia 2010; 58: 1905-19012

18. Bessler H, Djaldetti R, Salman H, Bergman M, Djaldetti M. IL-1 beta, IL-2, IL-6 and TNF-alpha production by peripheral blood mononuclear cells from patients with Parkinson's disease. Biomed Pharmacother 1999; 53: 141-145.

19. Dufek M, Rektorova I, Thon V, Lokaj J, Rektor I. Interleukin-6 may contribute to mortality in Parkinson's disease patients: a 4-year prospective study. Parkinsons Dis 2015; 2015: 898192

20. Lofrumento DD, Saponaro C, Cianciulli A, De Nuccio F, Mitolo V, Nicolardi G et al. MPTP-induced neuroinflammation increases the expression of pro-inflammatory cytokines and their receptors in mouse brain. Neuroimmunomodulation 2011; 18: 79-88.

21. Muller $T$, Blum-Degen D, Przuntek H, Kuhn W. Interleukin-6 levels in cerebrospinal fluid inversely correlate to severity of Parkinson's disease. Acta Neurol Scand 1998; 98: 142-144.

22. Spittau B, Zhou X, Ming M, Krieglstein K. IL6 protects MN9D cells and midbrain dopaminergic neurons from MPP+-induced neurodegeneration. Neuromol Med 2012; 14: 317-327.

23. Doorn KJ, Lucassen PJ, Boddeke HW, Prins M, Berendse HW, Drukarch B et al. Emerging roles of microglial activation and non-motor symptoms in Parkinson's disease. Prog Neurobiol 2012; 98: 222-238.

24. Tanaka S, Ishii A, Ohtaki H, Shioda S, Yoshida T, Numazawa S. Activation of microglia induces symptoms of Parkinson's disease in wild-type, but not in IL-1 knockout mice. J Neuroinflamm 2013; 10: 143.

25. Wilms H, Zecca L, Rosenstiel P, Sievers J, Deuschl G, Lucius R. Inflammation in Parkinson's diseases and other neurodegenerative diseases: cause and therapeutic implications. Curr Pharm Des 2007; 13: 1925-1928.

26. Chen T, Hou R, Xu S, Wu C. Donepezil regulates 1-methyl-4-phenylpyridinium-induced microglial polarization in Parkinson's disease. ACS Chem Neurosci 2015; 6: 1708-1714.

27. Shimma N, Akiyama N, Umezawa M, Okuma Y, Nomura Y, Saito T et al. Possible role of interleukin-6 in PC12 cell death induced by MPP ${ }^{+}$and tetrahydroisoquinoline. J Pharmacol Sci 2003; 93: 471-477.

28. Chakrabarty P, Ceballos-Diaz C, Lin WL, Beccard A, Jansen-West K, McFarland NR et al. Interferon- $\gamma$ induces progressive nigrostriatal degeneration and basal ganglia calcification. Nat Neurosci 2011; 14: 694-696.

29. Barcia C, Ros CM, Annese V, Gómez A, Ros-Bernal F, Aguado-Yera D et al. IFN- $\gamma$ signaling, with the synergistic contribution of TNF- $\alpha$, mediates cell specific microglial and astroglial activation in experimental models of Parkinson's disease. Cell Death Dis 2011; 2: e142.

30. Veldhoen M, Hocking RJ, Atkins CJ, Locksley RM, Stockinger B. TGF beta in the context of an inflammatory cytokine milieu supports de novo differentiation of IL-17-producing T cells. Immunity 2006; 24: 179-189.

31. Espejo EF, Gonzalez-Albo MC, Moraes JP, El Banoua F, Flores JA, Caraballo I. Functional regeneration in a rat Parkinson's model after intrastriatal grafts of glial cell line-derived neurotrophic factor and transforming growth factor beta1-expressing extra-adrenal chromaffin cells of the Zuckerkandl's organ. J Neurosci 2001; 21: 9888-9895.

32. Joniec-Maciejak I, Ciesielska A, Wawer A, Sznejder-Pachołek A, Schwenkgrub J, Cudna A et al. The influence of AAV2-mediated gene transfer of human IL-10 on neurodegeneration and immune response in a murine model of Parkinson's disease. Pharmacol Rep 2014; 66: 660-669.

33. Hirsch E, Graybiel AM, Agid YA. Melanized dopaminergic neurons are differentially susceptible to degeneration in Parkinson's disease. Nature 1988; 334: 345-348.

34. Finsterwald C, Magistretti PJ, Lengacher S. Astrocytes: new targets for the treatment of neurodegenerative diseases. Curr Pharm Des 2015; 21: 3570-3581. 
35. Radford RA, Morsch M, Rayner SL, Cole NJ, Pountney DL, Chung RS. The established and emerging roles of astrocytes and microglia in amyotrophic lateral sclerosis and frontotemporal dementia. Front Cell Neurosci 2015; 9: 414.

36. Verkhratsky A, Zorec R, Rodriguez JJ, Parpura V. Astroglia dynamics in ageing and Alzheimer's disease. Curr Opin Pharmacol 2016; 26: 74-79.

37. Verkhratsky A, Parpura V. Astrogliopathology in neurological, neurodevelopmental and psychiatric disorders. Neurobiol Dis 2016; 85: 254-261.

38. Jackson-Lewis V, Przedborski S. Protocol for the MPTP mouse model of Parkinson's disease. Nat Protocol 2007; 2: 141-151.

39. Tatton NA, Kish SJ. In situ detection of apoptotic nuclei in the substantia nigra compacta of 1-methyl-4-phenyl-1,2,3,6- tetrahydropyridine- treated mice using terminal deoxynucleotidyl transferase labelling and acridine orange staining. Neuroscience 1997; 77: 1037-1048.

40. Patil SP, Jain PD, Sancheti JS, Ghumatkar PJ, Tambe R, Sathaye S. Neuroprotective and neurotrophic effects of Apigenin and Luteolin in MPTP induced parkinsonism in mice. Neuropharmacology 2014; 86: 192-202.

41. Muñoz-Manchado AB, Villadiego J, Romo-Madero S, Suárez-Luna N, Bermejo-Navas A, Rodríguez-Gómez JA. Chronic and progressive Parkinson's disease MPTP model in adult and aged mice. J Neurochem 2016; 136: 373-387.

42. Goldberg NR, Haack AK, Lim NS, Janson OK, Meshul CK. Dopaminergic and behavioral correlates of progressive lesioning of the nigrostriatal pathway with 1-methyl-4-phenyl1,2,3,6- tetrahydropyridine. Neuroscience 2011; 180: 256-271.

43. Smeyne RJ, Breckenridge CB, Beck M, Jiao Y, Butt MT, Wolf JC. Assessment of the effects of MPTP and paraquat on dopaminergic neurons and microglia in the substantia nigra pars compacta of C57BL/6 mice. PLoS One 2016; 11: e0164094.

44. Bayer Andersen K, Leander Johansen J, Hentzer M, Smith GP, Dietz GP. Protection of primary dopaminergic midbrain neurons by GPR139 agonists supports different mechanisms of $\mathrm{MPP}^{+}$and rotenone toxicity. Front Cell Neurosci 2016; 10: 164.

45. Jaumotte JD, Wyrostek SL, Zigmond MJ. Protection of cultured dopamine neurons from $\mathrm{MPP}^{+}$requires a combination of neurotrophic factors. Eur J Neurosci 2016; 44: 1691-1699.

46. Zhang $Y$, Zhang $X$, Qu S. Ceftriaxone protects astrocytes from $M P P(+)$ via suppression of NF-kappaB/JNK/c-Jun signaling. Mol Neurobiol 2015; 52: 78-92.

47. Yu S, Wang X, He X, Wang Y, Gao S, Ren L et al. Curcumin exerts anti-inflammatory and antioxidative properties in 1-methyl-4-phenylpyridiniumion $\left(\mathrm{MPP}^{+}\right)$-stimulated mesencephalic astrocytes by interference with TLR4 and downstream signaling pathway. Cell Stress Chaperones 2016; 21: 697-705.

48. Liu K, Shi N, Sun Y, Zhang T, Sun X. Therapeutic effects of rapamycin on MPTP-induced Parkinsonism in mice. Neurochem Res 2013; 38: 201-207.

49. Aoyama K, Matsumura N, Watabe M, Nakaki T. Oxidative stress on EAAC1 is involved in MPTP-induced glutathione depletion and motor dysfunction. Eur J Neurosci 2008; 27: 20-30

50. Assous M, Had-Aissouni L, Gubellini P, Melon C, Nafia I, Salin P et al. Progressive Parkinsonism by acute dysfunction of excitatory amino acid transporters in the rat substantia nigra. Neurobiol Dis 2014; 65: 69-81.

51. Chung EK, Chen LW, ChanYS, Yung KK. Downregulation of glial glutamate transporters after dopamine denervation in the striatum of 6-hydroxydopamine-lesioned rats. J Comp Neurol 2008; 511: 421-437.

52. Moriguchi S, Yabuki Y, Fukunaga K. Reduced calcium/calmodulin-dependent protein kinase II activity in the hippocampus is associated with impaired cognitive function in MPTP-treated mice. J Neurochem 2012; 120: 541-551.

53. Pita-Almenar JD, Zou S, Colbert CM, Eskin A. Relationship between increase in astrocytic GLT-1 glutamate transport and late-LTP. Learn Mem 2012; 19: 615-626.
54. Boehmer C, Henke G, Schniepp R, Palmada M, Rothstein JD, Bröer S et al. Regulation of the glutamate transporter EAAT1 by the ubiquitin ligase Nedd4-2 and the serum and glucocorticoid-inducible kinase isoforms SGK1/3 and protein kinase B. J Neurochem 2003; 86: 1181-1188.

55. Boehmer C, Palmada M, Rajamanickam J, Schniepp R, Amara S, Lang F. Post-translational regulation of EAAT2 function by co-expressed ubiquitin ligase Nedd4-2 is impacted by SGK kinases. J Neurochem 2006; 97: 911-921.

56. García-Tardón N, González-González IM, Martínez-Villarreal J, Fernández-Sánchez $E$, Giménez C, Zafra F. Protein kinase C (PKC)-promoted endocytosis of glutamate transporter GLT-1 requires ubiquitin ligase Nedd4-2-dependent ubiquitination but not phosphorylation. J Biol Chem 2012; 287: 19177-19187.

57. Bolin LM, Strycharska-Orczyk I, Murray R, Langston JW, Di Monte D. Increased vulnerability of dopaminergic neurons in MPTP-lesioned interleukin-6 deficient mice. J Neurochem 2002 83: $167-175$.

58. Li XZ, Bai LM, Yang YP, Luo WF, Hu WD, Chen JP et al. Effects of IL-6 secreted from astrocytes on the survival of dopaminergic neurons in lipopolysaccharide-induced inflammation. Neurosci Res 2009; 65: 252-258.

59. Ji YF, Zhou L, Xie YJ, Xu SM, Zhu J, Teng P et al. Upregulation of glutamate transporter GLT-1 by mTOR-Akt-NF-kB cascade in astrocytic oxygen-glucose deprivation. Glia 2013; 61: 1959-1975.

60. Wu X, Kihara T, Akaike A, Niidome T, Sugimoto H. PI3K/Akt/mTOR signaling regulates glutamate transporter 1 in astrocytes. Biochem Biophys Res Commun 2010; 393: 514-518.

61. Feng D, Guo B, Liu G, Wang B, Wang W, Gao G et al. FGF2 alleviates PTSD symptoms in rats by restoring GLAST function in astrocytes via the JAK/STAT pathway. Eur Neuropsychopharmacol 2015; 25: 1287-1299.

62. Hung CC, Lin $\mathrm{CH}$, Chang H, Wang $\mathrm{CY}$, Lin SH, Hsu PC et al. Astrocytic GAP43 induced by the TLR4/NF-KB/STAT3 axis attenuates astrogliosis-mediated microglial activation and neurotoxicity. J Neurosci 2016; 36: 2027-2043.

63. Sundar BS, Barbara VM, Roemgens A, Beyer C, Arnold S. Sex- and brain region-specific role of cytochrome $\mathrm{C}$ oxidase in 1-methyl-4-phenylpyridinium-mediated astrocyte vulnerability. J Neurosci Res 2011; 89: 2068-2082.

64. Chen PC, Vargas MR, Pani AK, Smeyne RJ, Johnson DA, Kan YW et al. Nrf2-mediated neuroprotection in the MPTP mouse model of Parkinson's disease: critical role for the astrocyte. Proc Natl Acad Sci USA 2009; 106: 2933-2938.

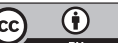

Cell Death and Disease is an open-access journal published by Nature Publishing Group. This work is licensed under a Creative Commons Attribution 4.0 International License. The images or other third party material in this article are included in the article's Creative Commons license, unless indicated otherwise in the credit line; if the material is not included under the Creative Commons license, users will need to obtain permission from the license holder to reproduce the material. To view a copy of this license, visit http://creativecommons.org/licenses/by/4.0/

C) The Author(s) 2017

Supplementary Information accompanies this paper on Cell Death and Disease website (http://www.nature.com/cddis) 